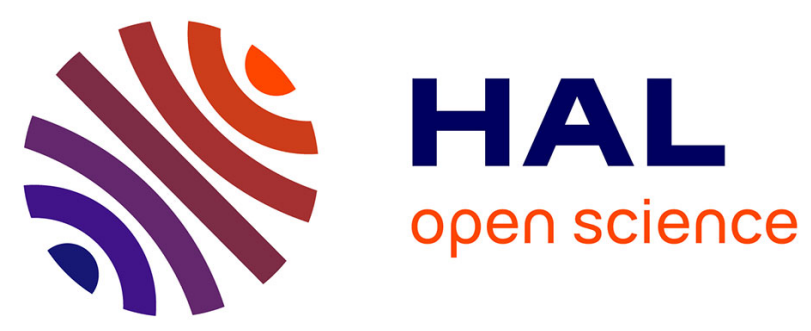

\title{
A sparse preconditioned iterative method for vibration analysis of geometrically mistuned bladed disks
}

Vladislav Ganine, Mathias Legrand, Hannah Michalska, Christophe Pierre

\section{To cite this version:}

Vladislav Ganine, Mathias Legrand, Hannah Michalska, Christophe Pierre. A sparse preconditioned iterative method for vibration analysis of geometrically mistuned bladed disks. Computers \& Structures, 2009, 87 (5-6), pp.342-354. 10.1016/j.compstruc.2008.12.011 . hal-00363296

\section{HAL Id: hal-00363296 \\ https://hal.science/hal-00363296}

Submitted on 21 Feb 2009

HAL is a multi-disciplinary open access archive for the deposit and dissemination of scientific research documents, whether they are published or not. The documents may come from teaching and research institutions in France or abroad, or from public or private research centers.
L'archive ouverte pluridisciplinaire HAL, est destinée au dépôt et à la diffusion de documents scientifiques de niveau recherche, publiés ou non, émanant des établissements d'enseignement et de recherche français ou étrangers, des laboratoires publics ou privés. 


\title{
A sparse preconditioned iterative method for vibration analysis of geometrically mistuned bladed disks
}

\author{
Vladislav Ganine ${ }^{1}$ \\ Structural Dynamics and Vibration Laboratory, Department of Mechanical Engineering, McGill \\ University, 817 Sherbrooke St West, Montreal, Quebec H3A 2K6, Canada \\ Mathias Legrand \\ Structural Dynamics and Vibration Laboratory, Department of Mechanical Engineering, McGill \\ University, 817 Sherbrooke St West, Montreal, Quebec H3A 2K6, Canada
}

\section{Hannah Michalska}

Department of Electrical and Computer Engineering, McGill University, Montreal, Quebec H3A 2A7, Canada

\section{Christophe Pierre}

Structural Dynamics and Vibration Laboratory, Department of Mechanical Engineering, McGill University, 817 Sherbrooke St West, Montreal, Quebec H3A 2K6, Canada

\begin{abstract}
In this paper, the Static Mode Compensation method to predict geometrical mistuning effects on the response of bladed disks is reviewed and its limitations are analyzed. This method proved to be effective only for narrow clusters of modes under localized low rank perturbation. A new algorithm is introduced to address its deficiencies that draws on optimal preconditioned methods for generalized eigenvalue problem featuring sparse matrix vector multiplications, being more attractive under limited memory constraints of multi-millon DOF FEM models. The central idea of the SMC is to correct nominal eigenspace using modal acceleration method. It has been extended here by replacing the quasi-static set of modes with inexact solutions of the linear Jacobi-Davidson correction equations. Some heuristic strategies are discussed to lower the computational effort given the block-circulant structure of the nominal system. Numerical experiments on an industrial scale bladed disk model show that this leads to a very competitive tool. Computational performance and accuracy of both methods is compared in two areas of spectrum. The study demonstrates low accuracy of SMC method in the modal interaction zone, while validating efficiency and accuracy of the new algorithm in both areas.
\end{abstract}

Keywords: Mistuning Turbomachinery Model reduction Indirect methods

\section{Motivation and overview}

Bladed disk assemblies belong to a class of rotationally periodic systems in which cyclic symmetry is commonly exploited to predict vibrational response because it reduces the size of a problem to a single sector [2]. However, in practice the symmetry is destroyed by unavoidable blade-to-blade properties variations due to manufacturing tolerances and operational wear and tear, known in the industry as structural mistuning problem. These imperfections could cause large increases in stress

\footnotetext{
${ }^{1}$ Corresponding author: vladislav.ganine@mail.mcgill.ca
} 
and vibration amplitudes with respect to the nominal condition. Therefore computationally effective methods to predict and quantify the response amplitudes of mistuned rotors are of great interest to the manufacturers of turbine engines.

There exists a significant body of work where mistuned rotor forced response prediction tools are built from parent FE (Finite Element) models of order $n$ using reduced models of order $m \ll$ $n[1,2,3]$. Most of the reduction methods for bladed rotors involve projection into a lower order subspace spanned by a small number of nominal system modes corresponding to a contiguous set of eigenvalues in the frequency band of interest. Note that the projection is done on a subspace of the same dimension $m$ as the number of eigenvectors one wants to approximate. Extraction of approximations of perturbed eigenpairs $\left(\widehat{\lambda}_{i}, \widehat{\mathbf{x}}_{i}\right), i=1, \ldots, m$ from a low-order subspace $\mathscr{X} \in \mathbb{R}^{m}$ is essentially the Rayleigh-Ritz procedure. Let $(\mathbf{A}, \mathbf{B})$ correspond to nominal symmetric indefinite stiffness and symmetric positive definite mass matrices with $(\Delta \mathbf{A}, \Delta \mathbf{B})$ denoting perturbations to these matrices due to mistuning. In its practical less expensive form (if $(\mathbf{B}+\Delta \mathbf{B})$-orthonormalization of $\mathbf{X}$ is omitted) the procedure leads to a projected generalized eigenvalue problem and goes as follows:

1. Let the set of nominal modes $\mathbf{X}$ form a basis of $\mathscr{X}$.

2. Compute $\mathbf{H}=\mathbf{X}^{\mathrm{T}}(\mathbf{A}+\Delta \mathbf{A}) \mathbf{X}$ and $\mathbf{G}=\mathbf{X}^{\mathrm{T}}(\mathbf{B}+\Delta \mathbf{B}) \mathbf{X}$.

3. Find eigenpairs $\left(\mu_{i}, \mathbf{z}_{i}\right)$ of the matrix pair $(\mathbf{H}, \mathbf{G})$.

4. Accept $\left(\mu_{i}, \mathbf{X z}_{i}\right)$ as an approximate eigenpair $\left(\widehat{\lambda}_{i}, \widehat{\mathbf{x}}_{i}\right)$ of the matrix pair $(\mathbf{A}+\Delta \mathbf{A}, \mathbf{B}+\Delta \mathbf{B})$.

While effective, such approximation is limited in one basic assumption that the perturbation to nominal matrices do not significantly change the eigenspace from which we extract the perturbed eigenpairs. For an accurate approximation the angle between eigenvector $\widehat{\mathbf{x}}_{i}$ and subspace $\mathscr{X}$ must be sufficiently small. This assumption enables great computational efficiencies but clearly does not hold true for large magnitude perturbations such as geometric mistuning. The smaller angles can be achieved in two ways. One may build a search subspace $\mathscr{X}$ of higher dimension by including more nominal eigenvectors or even combine them within a domain decomposition method [4]. In practice, that results in subspaces of a very high-order rendering them computationally impractical. An alternative approach is to correct the set of nominal eigenvectors [5]. The SMC (Static Mode Compensation) method inspired by modal acceleration technique yields accurate approximates of perturbed eigenpairs under large geometric mistuning extracted from a low dimensional subspace.

Several other efforts can be mentioned in relation to dynamic response quantification of geometrically mistuned bladed disks. Sinha [6] presented the MMDA (Modified Modal Domain Analysis) method for the large geometric mistuning problem, where he proposed to build a test subspace by including a small set of nominal eigenvectors of several unperturbed periodic systems, one for each POD feature. Petrov et al. [7] used the Sherman-Morrison-Woodbury formula to calculate the perturbed FRF (Frequency Response Function) inverse. Yet that algorithm does not compute mistuned system modes, the vibration response is estimated for a limited number of DOF.

The main goal of this paper is to analyze the Static Mode Compensation method by taking the viewpoint of the perturbed generalized eigenvalue problem as a nonlinear system of equations. We consider the cases for which the method should work reasonably well, in situations where it would not fit, we propose a more sophisticated algorithm to correct nominal modes by adopting a Newtontype framework. The Jacobi-Davidson scheme [8], the Trace Minimization method [9] and a number of related algorithms all fall in that category. Our algorithm is essentially an adaptation of the Jacobi-Davidson scheme to the block-circulant system under a limited class of perturbations. We will see that since the algorithm is based on an iterative linear solver, it is more memory efficient and independent from the structure of perturbation as compared to SMC. With the selected class of perturbations the method should converge significantly faster to a target eigenspace than any general purpose iterative eigensolver that does not exploit the structure of the system. 
The paper is organized as follows. SMC algorithm approximation properties and ties with other methods are studied in section 2. A new algorithm based on the Jacobi-Davidson framework is described in section 3 . In section 4 , convincing numerical evidence of relative performance and accuracy of both algorithms is given using an industrial bladed disk FEM. In particular, the foreign object ingestion event is considered for two different areas of spectrum. Conclusion is given in section 5 .

\section{SMC analysis}

Throughout this paper we consider the harmonic steady state response of an undamped mistuned bladed disk finite element model. In the absence of excitation in frequency domain the equation of motion can be written as:

$$
\left((\mathbf{A}+\Delta \mathbf{A})-\widehat{\lambda}_{i}(\mathbf{B}+\Delta \mathbf{B})\right) \widehat{\mathbf{x}}_{i}=0
$$

where $\widehat{\mathbf{x}_{i}}$ is $i$ th mistuned mode, $\sqrt{\widehat{\lambda}_{i}}$ is $i$ th natural frequency, (A, B) are real symmetric block-circulant nominal mass and stiffness matrices, $\mathbf{A}$ nonnegative definite and $\mathbf{B}$ positive definite, $(\Delta \mathbf{A}, \Delta \mathbf{B})$ are symmetric perturbations due to geometric mistuning with the sparsity pattern (zero entries structure) $\mathscr{S}(\Delta \mathbf{A}, \Delta \mathbf{B}) \subset \mathscr{S}(\mathbf{A}, \mathbf{B})$. For parts of the discussion we will make assumptions on the class of perturbations owing to the special structure of the nominal system. It is natural to consider the perturbation matrices due to geometric mistuning structured as subset of nominal symmetric blockcirculant structure. If we denote any vector norm and corresponding subordinate matrix norm as $\|\cdot\|$, the perturbations satisfying $\|\Delta \mathrm{A}\|<\epsilon\|\mathrm{A}\|$ for small enough $\epsilon$ are referred to as low magnitude perturbations. The influence of the structure of perturbation on the behavior of perturbed eigenpairs is reflected by the rank. Thus $\Delta \mathrm{A}$ localized to one or few blocks of a block-circulant structure with $\operatorname{rank}\{\Delta A\} \ll \operatorname{rank}\{A\}$ is referred to as low rank localized perturbation.

The main idea behind the SMC method, originally presented in Lim et al. [5], is based on the classical MAM (Modal Acceleration Method) used to reduce the modal truncation error of an expanded in modal space FRF. Consider the frequency response of the mistuned system expanded in the truncated modal space under arbitrary excitation $\mathbf{f}(\omega)$ :

$$
\mathbf{x}(\omega)=\sum_{i=1}^{m} \frac{\widehat{\mathbf{x}}_{i}^{\mathrm{T}} \mathbf{f}(\omega)}{\widehat{\lambda}_{i}-\omega^{2}} \widehat{\mathbf{x}}_{i}
$$

If we apply MAM it becomes:

$$
\mathbf{x}(\omega)=\left((\mathbf{A}+\Delta \mathbf{A})-\omega_{c}{ }^{2}(\mathbf{B}+\Delta \mathbf{B})\right)^{-1} \mathbf{f}(\omega)+\sum_{i=1}^{m}\left(\frac{\omega^{2}-\omega_{c}{ }^{2}}{\widehat{\lambda}_{i}-\omega_{c}{ }^{2}}\right) \frac{\widehat{\mathbf{x}}_{i}^{\mathrm{T}} \mathbf{f}(\omega)}{\widehat{\lambda}_{i}-\omega^{2}} \widehat{\mathbf{x}}_{i}
$$

where the eigenvalue shift is usually selected in the middle of the ROM (Reduced Order Model) frequency band $\omega_{c}^{2}=0.5\left(\widehat{\lambda}_{1}+\widehat{\lambda}_{m}\right)$, or zero if low frequency modes are not truncated. Lim proposed to use the first term on the right hand side to correct the set of system nominal mode shapes $\mathbf{x}_{j}$ such that they approximately span the same subspace as the perturbed eigenvectors $\widehat{\mathbf{x}}_{i}$ :

$$
\mathbf{x}_{j}\left(\omega_{j}\right)-\Delta \mathbf{x}_{j}=\sum_{i=1}^{m}\left(\frac{\omega_{j}^{2}-\omega_{c}^{2}}{\widehat{\lambda}_{i}-\omega_{c}^{2}}\right) \frac{\widehat{\mathbf{x}}_{i}^{\mathrm{T}} \mathbf{f}\left(\omega_{j}\right)}{\widehat{\lambda}_{i}-\omega_{j}^{2}} \widehat{\mathbf{x}}_{i}
$$

where the correction terms $\Delta \mathbf{x}_{j}$ called quasi-static modes are:

$$
\Delta \mathbf{x}_{j}=\left((\mathbf{A}+\Delta \mathbf{A})-\omega_{c}^{2}(\mathbf{B}+\Delta \mathbf{B})\right)^{-1} \mathbf{f}\left(\omega_{j}\right)
$$


Notice that in this setting $\omega_{j}^{2}$ corresponds to a nominal system eigenvalue $\lambda_{j}$, while $\mathbf{f}\left(\omega_{j}\right)$ is an equivalent to geometric mistuning forcing excitation such that the motion of perturbed system excited at each of the unperturbed natural frequencies corresponds to the nominal eigenvector $\mathbf{x}_{j}$ at that frequency:

$$
\mathbf{f}\left(\omega_{j}\right)=\left((\mathbf{A}+\Delta \mathbf{A})-\lambda_{j}(\mathbf{B}+\Delta \mathbf{B})\right) \mathbf{x}_{j}=\left(\Delta \mathbf{A}-\lambda_{j} \Delta \mathbf{B}\right) \mathbf{x}_{j}
$$

Rather than performing matrix inversion of system order, $\Delta \mathbf{x}_{j}$ are computed by exploiting the zero structure of perturbation terms $(\Delta \mathbf{A}, \Delta \mathbf{B})$ as well as cyclic symmetry of the nominal matrix pair $(\mathbf{A}, \mathbf{B})$. Numerical evidence shows that a straightforward application of the SMC algorithm is not always obvious or even adequate for some very large order models, classes of perturbation and areas of spectrum, unless its convergence properties and other limitations are well understood. First, the algorithm implies the use of fully populated matrices $\left(\mathbf{A}-\omega_{c}{ }^{2} \mathbf{B}\right)^{-1}$, this can be done efficiently memorywise only if the rank of perturbation and the order of the system is sufficiently low. An optimal choice of $\omega_{c}$ also remains to be an issue since it must not cross the nominal and perturbed system natural frequencies, with the latter unknown a priori, while its effect on convergence properties is yet unclear. The primary reason for calculation of correction terms is generation of very compact yet accurate ROM. However the latter is not always the case even for small rank perturbations affecting limited number of blades. If a simple strategy [10] of augmenting the projection basis by including more corrected nominal modes is adopted to increase the accuracy, it may lead to a very slow convergence while generating high dimensional test subspaces.

In order to better understand the convergence properties of SMC we adopt the viewpoint taken by many subspace-based methods for computing eigenvectors and eigenvalues of large sparse matrices. Assume that we have a set of nominal eigenvectors and corresponding eigenvalues $\left(\lambda_{i}, \mathbf{x}_{i}\right)$ that only approximate eigenpairs $\left(\widehat{\lambda}_{i}, \widehat{\mathbf{x}}_{i}\right)$ of the mistuned matrix pair $(\widehat{\mathbf{A}}, \widehat{\mathbf{B}})$. In order to find a way to correct a given approximate eigenpair the generalized eigenvalue problem can be viewed as a nonlinear system of equations:

$$
\left(\widehat{\mathbf{A}}-\widehat{\lambda_{i}} \widehat{\mathbf{B}}\right) \widehat{\mathbf{x}}_{i}=0
$$

It is a system of $n$ equations with $n+1$ unknowns, so a constraint should be imposed: usually the eigenvectors are mass-orthonormalized $\left\|\widehat{\mathbf{x}}_{i}\right\|_{\widehat{\mathbf{B}}}=1$, where the norm $\|\cdot\|_{\widehat{\mathbf{B}}}$ is defined in the inner product space $(\mathbf{x}, \mathbf{y})_{\widehat{\mathbf{B}}}=\mathbf{x}^{\mathrm{T}} \widehat{\mathbf{B}} \mathbf{y}$. Given an approximate nominal eigenpair $\left(\lambda_{i}, \mathbf{x}_{i}\right)$ find a correction $\left(\Delta \lambda_{i}, \Delta \mathbf{x}_{i}\right)$ to satisfy the system of nonlinear equations:

$$
\begin{aligned}
& \left(\left(\widehat{\mathbf{A}}-\lambda_{i} \widehat{\mathbf{B}}\right)-\Delta \lambda_{i} \widehat{\mathbf{B}}\right)\left(\mathbf{x}_{i}+\Delta \mathbf{x}_{i}\right)=0 \\
& \left\|\mathbf{x}_{i}+\Delta \mathbf{x}_{i}\right\|_{\widehat{\mathbf{B}}}=1
\end{aligned}
$$

which can be rewritten as:

$$
\begin{aligned}
& \left(\widehat{\mathbf{A}}-\lambda_{i} \widehat{\mathbf{B}}\right) \Delta \mathbf{x}_{i}=-\left(\Delta \mathbf{A}-\lambda_{i} \Delta \mathbf{B}\right) \mathbf{x}_{i}+\Delta \lambda_{i} \widehat{\mathbf{B}} \mathbf{x}_{i}+\Delta \lambda_{i} \widehat{\mathbf{B}} \Delta \mathbf{x}_{i} \\
& \left\|\mathbf{x}_{i}+\Delta \mathbf{x}_{i}\right\|_{\widehat{\mathbf{B}}}=1
\end{aligned}
$$

The correction terms are usually found by solving the linear system that results from omission of the nonlinear quadratic terms $\Delta \lambda_{i} \widehat{\mathbf{B}} \Delta \mathbf{x}_{i}$ as well as the terms $\Delta \lambda_{i} \widehat{\mathbf{B}} \mathbf{x}_{i}$ :

$$
\left(\widehat{\mathbf{A}}-\lambda_{i} \widehat{\mathbf{B}}\right) \Delta \mathbf{x}_{i}=-\left(\Delta \mathbf{A}-\lambda_{i} \Delta \mathbf{B}\right) \mathbf{x}_{i}
$$

Thus the generalized Davidson method [11] solves the resulting linear system for each eigenvector $\mathbf{x}_{i}$ as:

$$
\Delta \mathbf{x}_{i}=-\mathbf{K}^{-1}\left(\Delta \mathbf{A}-\lambda_{i} \Delta \mathbf{B}\right) \mathbf{x}_{i}
$$


with the help of preconditioner $\mathbf{K}^{-1}$ that approximates the inverse $\left(\widehat{\mathbf{A}}-\lambda_{i} \widehat{\mathbf{B}}\right)^{-1}$. It is easy to see that the SMC algorithm is equivalent to one iteration of the generalized Davidson method without subspace acceleration, where the same preconditioner $\mathbf{K}^{-1}=\left(\widehat{\mathbf{A}}-\omega_{c}{ }^{2} \widehat{\mathbf{B}}\right)^{-1}$ is applied for all corrected eigenvectors. Although qualitative convergence to external eigenpairs analysis of the Davidson method have been developed, the quantitative results seem more difficult to obtain. Still the following remarks are of interest to understanding in which situations SMC method will work. First observe that the omission of the quadratic term is valid only if $\mathbf{x}_{i}$ is close to an eigenvector $\widehat{\mathbf{x}}_{i}$ and there exists a small in norm solution to the original nonlinear equation $\Delta \mathbf{x}_{i}$, such that the quadratic term in it will have relatively small to no influence.

Notice also that the quality of preconditioner $\left(\widehat{\mathbf{A}}-\omega_{c}{ }^{2} \widehat{\mathbf{B}}\right)^{-1}$ depends on how close $\omega_{c}{ }^{2}$ is to currently approximated $\hat{\lambda}_{i}$. Suppose that we already know $\widehat{\lambda}_{i}=\lambda_{i}$, then both neglected terms in (9) would disappear due to $\Delta \lambda_{i}=0$ and the equation solved with the ideal preconditioner, the pseudoinverse $\left(\widehat{\mathbf{A}}-\lambda_{i} \widehat{\mathbf{B}}\right)^{\dagger}$, would give us the exact correction $\Delta \mathbf{x}_{i}$ to an approximate eigenvector $\mathbf{x}_{i}$. Therefore the correction happens mainly in the direction of the perturbed eigenvector with eigenvalue closest to $\omega_{c}{ }^{2}$, which is also evident if we apply the amplification factor line of thought presented in the following. Practically, should the linearization condition be satisfied, the precision of SMC is acceptable if we either select very narrow bands of nominal eigenpairs to correct or can provide a guess on the area of spectrum where the perturbed eigenvectors with larger angles to nominal eigenspace are most likely to occur, so that their corresponding eigenvalues would fall close enough to $\omega_{c}^{2}$.

\section{Jacobi-Davidson method for geometrical mistuning problem}

The Jacobi-Davidson algorithm, as originally developed by Sleijpen and Van der Vorst in [8], is an alternative more robust approach to calculate correction to a current approximation of an eigenvector that addresses the above-mentioned potential weaknesses of SMC. First, unlike Davidson method, its convergence is guaranteed whenever non-diagonal and non-positive definite preconditioners are used, which is often the case when we approximate the interior of the spectrum. Second, it avoids illconditioning of the linear correction equations when we cross the perturbed or nominal eigenvalues and we are not constrained with the choice of $\omega_{c}$. Moreover, the correction equations can be solved only approximately using an iterative solver such that no fully populated matrices are involved. At the same time controlling the number of inner iterations (the number of iterations of linear solver) can also be used to compensate for lower quality preconditioning. The algorithm goes as follows. After omitting the second-order term in (9), i.e. linearizing around $\Delta \mathbf{x}_{i}=0$, the correction equation becomes:

$$
\begin{aligned}
& \left(\widehat{\mathbf{A}}-\lambda_{i} \widehat{\mathbf{B}}\right) \Delta \mathbf{x}_{i}=-\mathbf{r}_{i}+\Delta \lambda_{i} \widehat{\mathbf{B}} \mathbf{x}_{i} \\
& \left\|\mathbf{x}_{i}+\Delta \mathbf{x}_{i}\right\|_{\widehat{\mathbf{B}}}=1
\end{aligned}
$$

where $\mathbf{r}_{i}$ denotes the residual vector $\left(\Delta \mathbf{A}-\lambda_{i} \Delta \mathbf{B}\right) \mathbf{x}_{i}$. It is a $n+1$ system of equations with $n+1$ unknowns that can be solved on a smaller subspace by invoking an orthogonal projector operator $\mathbf{P}=\left(\mathbf{I}-\mathbf{x}_{i} \mathbf{x}_{i}^{\mathrm{T}} \widehat{\mathbf{B}}\right)$. Observing that $\mathbf{P} \mathbf{x}_{i}=0$, we obtain a degenerate system:

$$
\mathbf{P}^{\mathrm{T}}\left(\widehat{\mathbf{A}}-\lambda_{i} \widehat{\mathbf{B}}\right) \Delta \mathbf{x}_{i}=-\mathbf{P}^{\mathrm{T}} \mathbf{r}_{i}
$$

Among all the solutions we seek one $\widehat{\mathbf{B}}$-orthogonal to $\mathbf{x}_{i}$, i.e. $\Delta \mathbf{x}_{i}=\mathbf{P} \Delta \mathbf{x}_{i}$. That yields the linear Jacobi-Davidson correction equation to solve for each approximate eigenvector $\mathbf{x}_{i}$ :

$$
\begin{aligned}
& \mathbf{P}^{\mathrm{T}}\left(\widehat{\mathbf{A}}-\lambda_{i} \widehat{\mathbf{B}}\right) \mathbf{P} \mathbf{z}_{i}=-\mathbf{P}^{\mathrm{T}} \mathbf{r}_{i} \\
& \Delta \mathbf{x}_{i}=\mathbf{P} \mathbf{z}_{i}
\end{aligned}
$$


It can be solved only approximately using a matrix-free iterative method, usually (if not always) in combination with a projected preconditioner. Unlike SMC, the standard Jacobi-Davidson method makes use of subspace acceleration, i.e. the computed term rather than correcting current approximation of an eigenvector is used for expansion of the test subspace. Each step of subspace expansion, termed outer iteration, is preceded by solution of the correction equation, followed by RayleighRitz procedure, and so on up until convergence to an eigenpair. The original formulation of JacobiDavidson algorithm deals with approximation of individual eigenpairs. If more than one eigenpair is to be corrected at each outer iteration, a more restrictive correction $\Delta \mathbf{x}_{i}$ is usually used, namely the one $\widehat{\mathbf{B}}$-orthogonal to already converged eigenvectors $\mathbf{q}_{1}, \ldots, \mathbf{q}_{k}$ and/or to some clustering Ritz vectors:

$$
\begin{aligned}
& \left(\mathbf{I}-\mathbf{Q} \mathbf{Q}^{\mathrm{T}} \widehat{\mathbf{B}}\right)^{\mathrm{T}}\left(\widehat{\mathbf{A}}-\lambda_{i} \widehat{\mathbf{B}}\right)\left(\mathbf{I}-\mathbf{Q} \mathbf{Q}^{\mathrm{T}} \widehat{\mathbf{B}}\right) \mathbf{z}_{i}=-\left(\mathbf{I}-\mathbf{Q} \mathbf{Q}^{\mathrm{T}} \widehat{\mathbf{B}}\right)^{\mathrm{T}} \mathbf{r}_{i} \\
& \Delta \mathbf{x}_{i}=\left(\mathbf{I}-\mathbf{Q} \mathbf{Q}^{\mathrm{T}} \widehat{\mathbf{B}}\right) \mathbf{z}_{i}
\end{aligned}
$$

with $\mathbf{Q}=\left[\mathbf{q}_{1}, \ldots, \mathbf{q}_{k}, \mathbf{x}_{i}\right]$.

\subsection{Computational strategies}

A direct application of Jacobi-Davidson inner-outer iteration scheme to a periodic system featuring large geometric mistuning could hardly be worth the trouble compared with any modern subspacebased eigensolver if it were not for a host of a priori information available on the structure of the system and perturbation. In this section we propose some heuristic strategies to lower the computational cost of Jacobi-Davidson scheme relevant to typical industrial applications. Thus we consider a FOD (Foreign Object Damage) event or any kind of local defect to an industrial bladed disk [12] as a target application, which represents a low rank local perturbation of relatively high magnitude. Our arguments are illustrated numerically on a test case model. It is an integrally bladed rotor depicted in Fig. 1(a) featuring 29 blades used in the second stage of a compressor. The finite element model was constructed with standard linear brick elements with total 126,846 DOF. The foreign object damage scenario in which one blade suffers severe mistuning, with all other blades being tuned is introduced by significantly changing the blade geometry, as shown in Fig. 1(b). The natural fre-

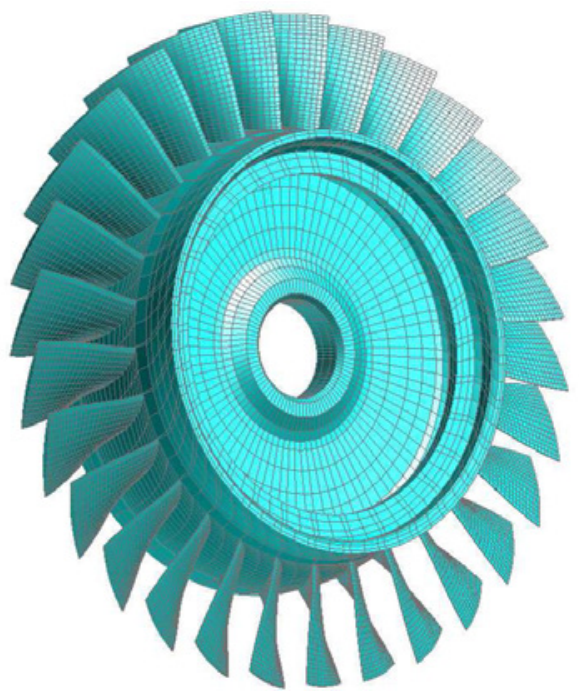

(a)

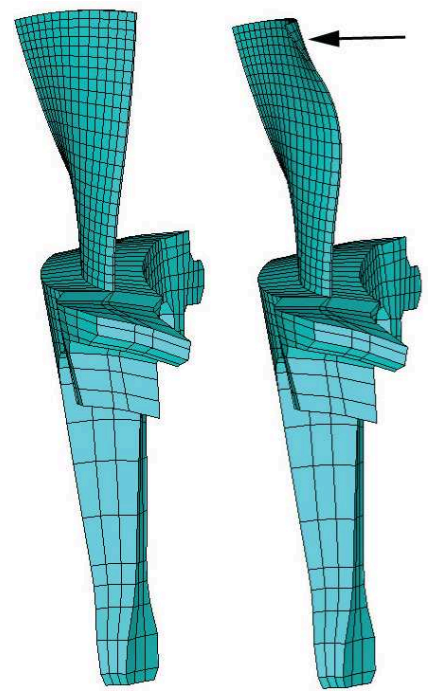

(b)

Figure 1: Finite element model of a integrally bladed rotor (a) and geometry of nominal and mistuned blades (b). The mistuning affects 1116 DOF of one blade. 
quencies and mode shapes of the nominal system are obtained via cyclic symmetry analysis using a finite element model of a single sector. Fig. 2 displays the free vibration natural frequencies of the tuned bladed disk versus the number of nodal diameters, where the frequency band of $34-36 \mathrm{kHz}$ corresponding to $2 \mathrm{~S}$ family of modes is chosen to illustrate the numerical strategies.

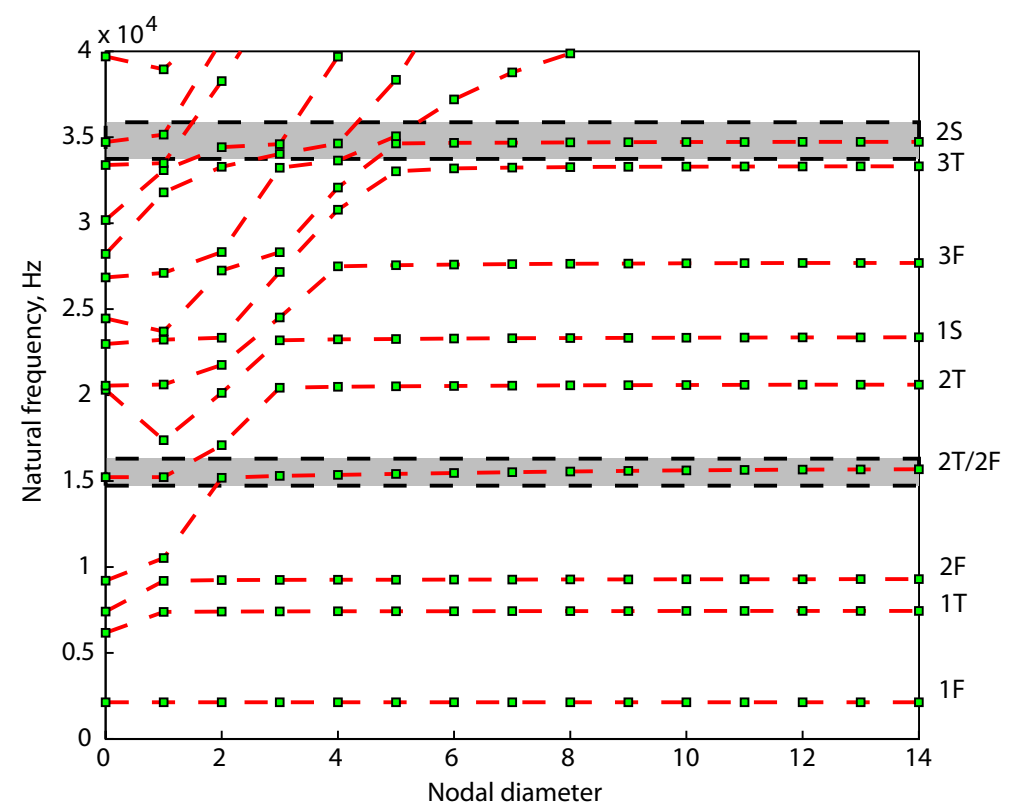

Figure 2: Natural frequencies versus nodal diameters. The frequency ranges that include $2 \mathrm{~S}$ and 2T/2F mode families are marked by horizontal lines.

\subsubsection{A priori information on block-circulant systems undergoing structured perturbation}

The effect of perturbation on the dynamics of periodic systems has been the subject of a number of theoretical studies [13, 14]. Although commonly the qualitative analysis is carried out on low-order simplified models of periodic systems, we can extend those observations to perturbed eigenstructure of very large scale periodic models. Typically, the eigenvectors of a periodic system are described in terms of nodal diameters (nodal lines across the diameter of a cyclic-symmetrical structure) and nodal circles (nodal lines in the circumferential direction). The number of nodal diameters for an eigenvector corresponds to a certain phase shift between adjacent blocks, given by:

$$
\alpha_{h}=\frac{2 \pi h}{\mathrm{~N}}
$$

where $h$ is the number of nodal diameters (or harmonic content of an eigenvector), and $\mathrm{N}$ is the number of blocks in the system. A periodic system also exhibits repeated natural frequencies for each harmonic, except $h=0$ and in the case of even N, except $h=\mathrm{N} / 2$, termed accordingly the doublet and singlet modes. Each member of a doublet has either sinusoidal or co-sinusoidal harmonic content, they are linearly independent, their absolute orientation is arbitrary due to symmetric nature of the system, while they can be distinguished by relative spatial phase shift. An interesting characteristic of periodic structures is the band structure of natural frequencies, that is the natural frequencies are grouped into narrow bands of mode families, in most cases forming well separated rather stable to perturbation eigenspaces. Since the test case model has no blade-to-blade shrouds, the blade motion dominated modes do not stiffen significantly as the number of nodal diameters increases, so they form lines that are approximately horizontal, which is shown in Fig. 2. 
When perturbation is introduced into a periodic system, the doublet mode pairs split such that each of the modes have a unique natural frequency. With low magnitude high rank perturbation the natural frequencies are still close in frequency, gradually splitting further apart as the magnitude increases. Low rank high magnitude perturbation limited to one or some blocks significantly affects only few natural frequencies. An example of frequency splitting phenomena for the test case model undergoing FOD event is shown in Fig. 3. The eigenvectors with introduction of moderate perturba-

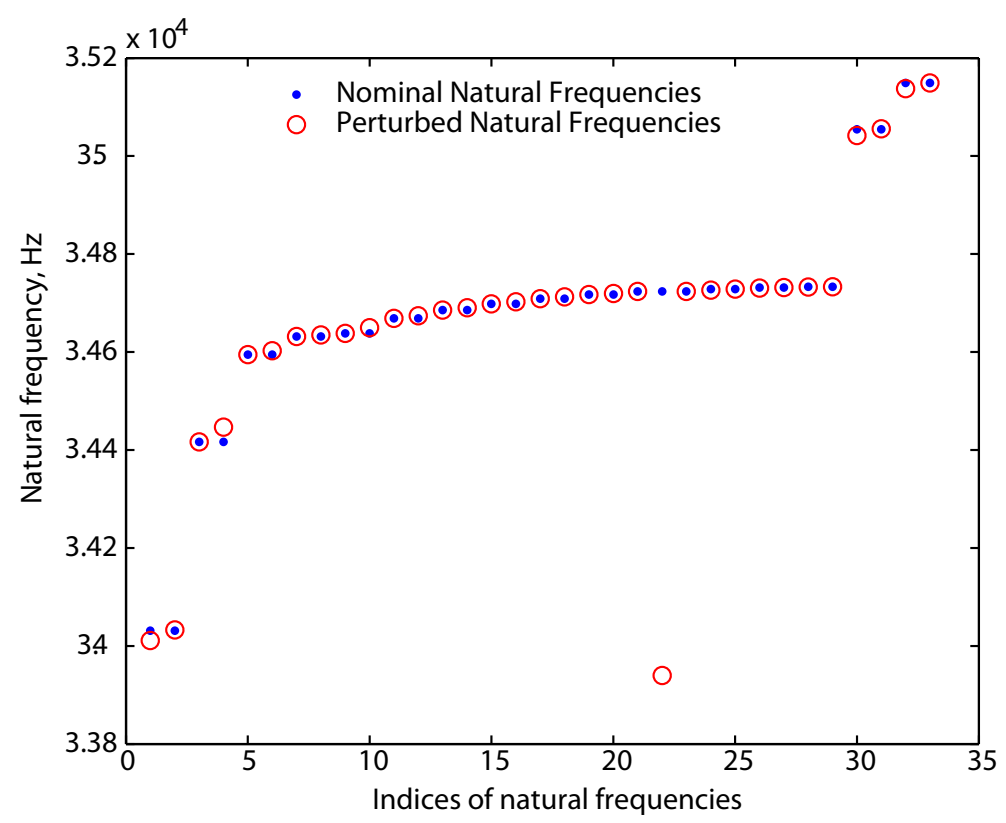

Figure 3: Nominal and perturbed natural frequencies for the test case model in $34-36 \mathrm{kHz}$ region. The perturbation brings about a localized mode with natural frequency far away from the unperturbed one. Otherwise, the clustered eigenvalues (all belong to $2 \mathrm{~S}$ family) seem to be more stable under perturbation.

tion would still be recognizable as ones of the nominal system. Although the perturbation destroys the regular features of mode shapes, they keep their original nodal diameter harmonic content, i.e. overall sinusoidal amplitude envelope, but transition from periodic to almost periodic [13]. As perturbation increases, the mode shapes sustain greater distortion until a sudden transition occurs, after which they become essentially localized around a single sector. However not all modes are equally sensitive to a particular perturbation, and not all of them experience sudden transitions, exhibiting rather a smoother transition from periodic to localized behavior. Fig. 4(a) shows the effect of low rank geometrical mistuning on the mode shapes for the test case model in $34-36 \mathrm{kHz}$ region. The mode distortion is reflected by the canonical angles. Note that the heavily distorted first mistuned mode forms large angles to any of the nominal modes, while one member of nearly each doublet is almost unaffected by perturbation. The combined effect of low-rank geometrical mistuning on an entire subspace spanned by nominal modes can be observed by calculating canonical angles between the corresponding eigenspaces, shown in Fig. 4(b). The perturbed eigenspace is very close to the nominal one, except for the five largest canonical angles introduced chiefly by five distorted modes with natural frequencies outside the main cluster, which is why the uncorrected nominal subspace cannot be used in Rayleigh-Ritz procedure. In addition to distortion of the original harmonic content in the mode shapes, perturbation also removes indeterminacy from the absolute orientation [14]. This phenomenon is illustrated in Fig. 5 where the norm of residual vector is calculated for the nominal modes subject to different spatial orientation. A nominal mode oriented to minimize the norm 


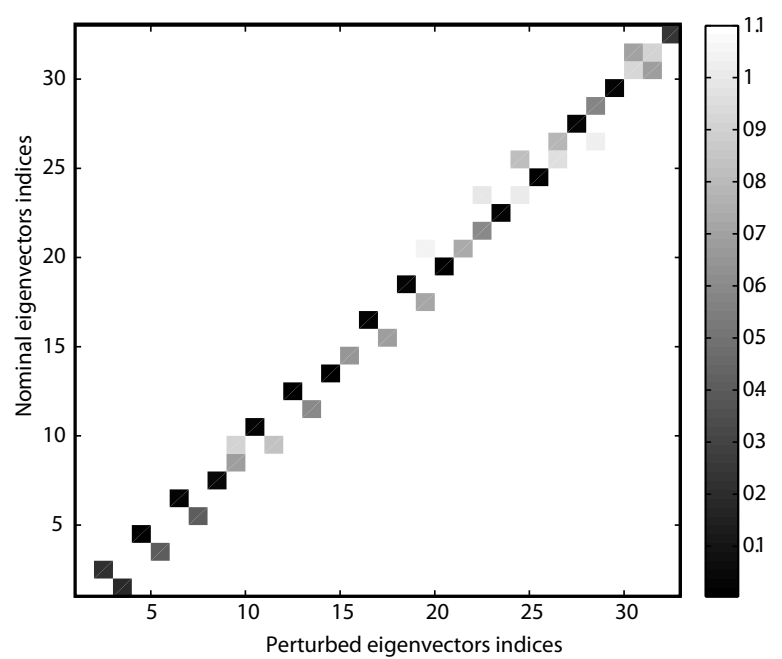

(a)

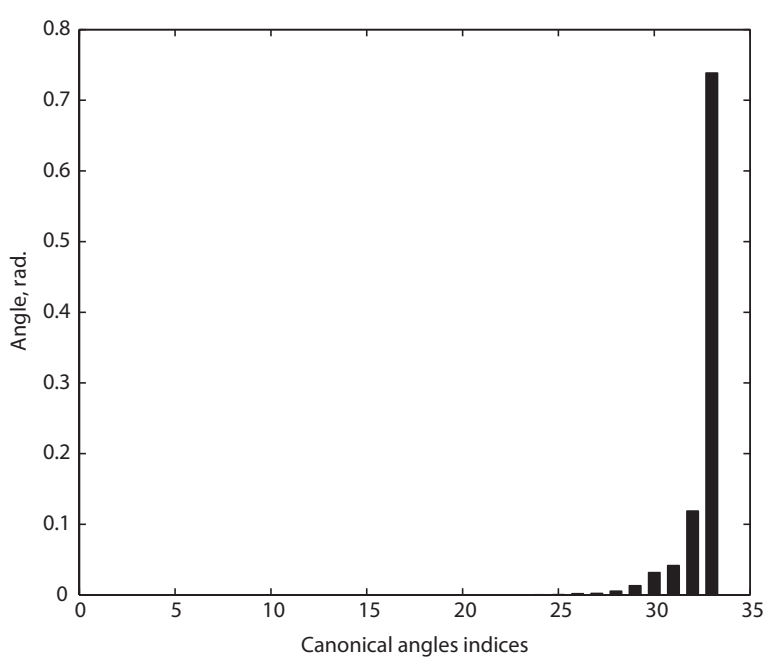

(b)

Figure 4: Canonical angles between nominal and perturbed individual eigenvectors (a) and eigenspaces (b) for the test case model in $34-36 \mathrm{kHz}$ region. Note the large angle that makes the first "rogue" localized perturbed mode with any of nominal ones. Also note that one member of almost every nominal doublet keeps its original harmonic content. Plot (b) shows the distance between perturbed and nominal eigenspaces. In order to extract accurate eigenpairs from the nominal eigenspace correction for the five largest canonical angles must be carried out.

would correspond to its perturbed almost periodic counterpart.

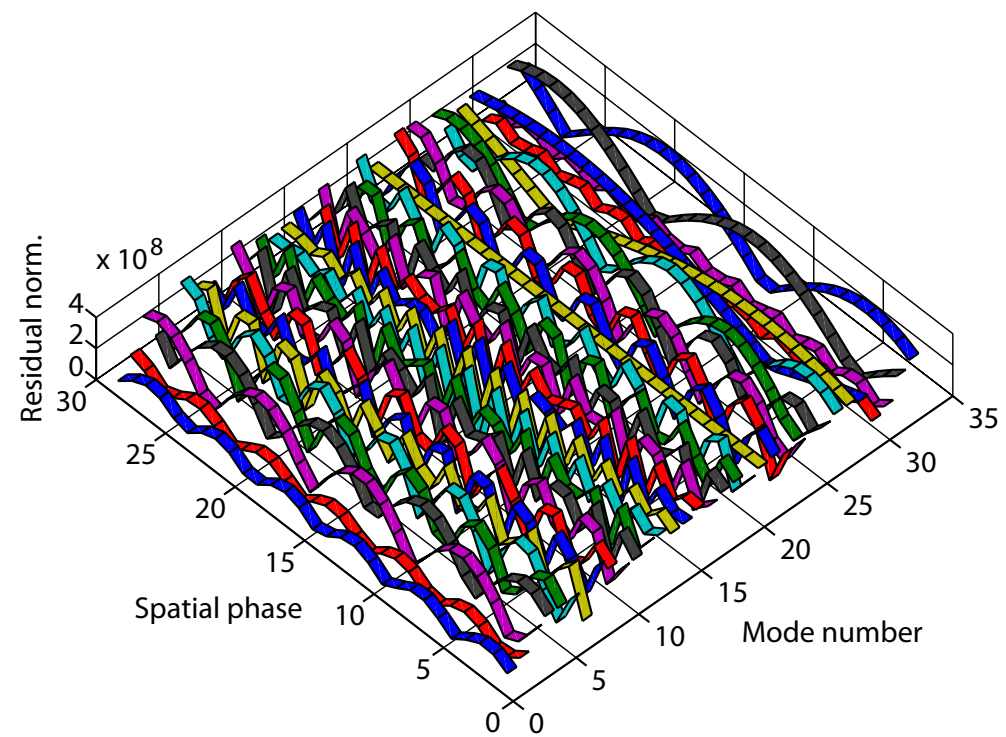

Figure 5: Norm of residual vectors calculated for nominal mode shapes in $34-36 \mathrm{kHz}$ region as a function of spatial orientation. The residual norm demonstrates arbitrary orientation and spatial periodicity of nominal eigenvectors. An oriented nominal eigenvector with minimum residual corresponds to its almost periodic perturbed counterpart. 


\subsubsection{Decreasing the number of correction equations to solve}

One of our goals in designing a practical reduction technique featuring large mistuning is computational efficiency, and since the major part of computational effort of the Jacobi-Davidson method is spent on solving the correction equations, we must find a way to decrease it. Observe that JD computes an orthogonal correction to an eigenvector that minimizes its residual vector by solving the linear equation (10). As such, JD correction term is designed to point in the direction of the closest perturbed eigenvector, the one that makes smallest angle with the current approximate eigenvector. Therefore, we can easily avoid computing corrections to both members of a doublet because the corrected eigenvectors would routinely point in the direction of the perturbed eigenvector that keeps maximum of the original harmonic content making smaller angle with both unperturbed nominal eigenvectors. Combined with subspace acceleration, such strategy lowers the computational work almost in half during the first outer iteration without compromising the accuracy of following Rayleigh-Ritz approximation. Out of two members of a doublet we select one with smaller residual based on the following arguments. First, it is expected that we will spare extra matrix vector multiplications of an iterative solver starting with already smaller residual. Valuable observation on the secondary effect of the smaller residual choice are given in [15]. Consider the exact solution of equation (10):

$$
\begin{aligned}
& \Delta \mathbf{x}_{i}=\mathbf{x}_{i}-\epsilon\left(\widehat{\mathbf{A}}-\lambda_{i} \widehat{\mathbf{B}}\right)^{-1} \widehat{\mathbf{B}} \mathbf{x}_{i} \\
& \epsilon=\frac{\mathbf{x}_{i}^{\mathrm{T}} \widehat{\mathbf{B}} \mathbf{x}_{i}}{\mathbf{x}_{i}^{\mathrm{T}} \widehat{\mathbf{B}}\left(\widehat{\mathbf{A}}-\lambda_{i} \widehat{\mathbf{B}}\right)^{-1} \widehat{\mathbf{B}} \mathbf{x}_{i}}
\end{aligned}
$$

If we express $\mathbf{x}_{i}$ as a linear combination of $\widehat{\mathbf{x}}_{j}$ it follows that:

$$
\left(\widehat{\mathbf{A}}-\lambda_{i} \widehat{\mathbf{B}}\right)^{-1} \widehat{\mathbf{B}} \mathbf{x}_{i}=\sum_{j} \frac{\alpha_{j}}{\widehat{\lambda}_{j}-\lambda_{i}} \widehat{\mathbf{x}}_{j}
$$

Observe that eigenvector components corresponding to eigenvalues closer to $\lambda_{i}$ will be amplified more in $\left(\widehat{\mathbf{A}}-\lambda_{i} \widehat{\mathbf{B}}\right)^{-1} \widehat{\mathbf{B}} \mathbf{x}_{i}$ with amplification factors $1 /\left|\widehat{\lambda}_{j}-\lambda_{i}\right|$. It is straightforward to see that due to the $\widehat{\mathbf{B}}$-orthogonalization, as soon as $\mathbf{x}_{i}$ has large component in the direction of $\widehat{\mathbf{x}}_{j}$, i.e. smaller angle between them or ultimately smaller residual, the components in the direction of the next closest $\widehat{\mathbf{x}}_{j+1}$ become dominant. Consequently, the correction term computed to turn the first nominal eigenvector with smaller residual in the direction of $\widehat{\mathbf{x}}_{j}$, if used with subspace acceleration, will inevitably introduce more additional information in the direction of the second perturbed member of the doublet.

\subsubsection{Reduction of number of inner iterations}

In addition to decreasing the number of correction equations to solve, we can also better exploit the a priori information to reduce the residual norm before applying any iterative solver. In the view of the fact that perturbed modes of periodic systems acquire spatial orientation, as depicted in Fig. 5, we can inexpensively find it by computing several residual vectors per double mode. Fig. 6 shows the effect of finding spatial orientation on initial residual norm, while Fig. 7(a) depicts its effect on the residual convergence history of GMRES (Generalized Minimum Residual) linear solver with preconditioning.

The information on how to provide an initial guess vector for iterative solver can be obtained if we argue heuristically as follows. It is a well known fact that if an eigenvalue problem has a cluster of eigenvalues, then the corresponding eigenvectors will be extremely ill conditioned, such that even an insignificant perturbation can drastically change the eigenvectors, while spanned by them eigenspace will be relatively well determined and well conditioned [16]. In [17], Theorem 1 implies 


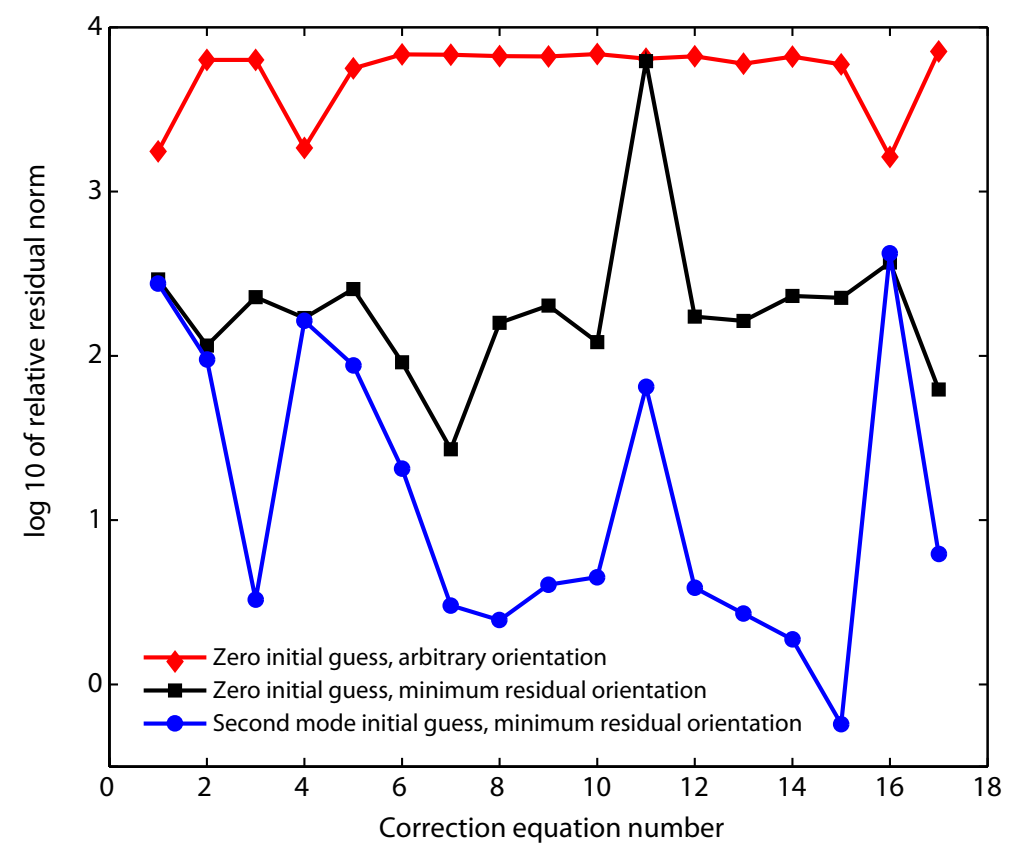

Figure 6: Effect of spatial orientation and initial guess vector on initial residual norm for the test case model in $34-36 \mathrm{kHz}$ region. By applying the knowledge about our system one can consistently reduce residual of the linear correction equation before any iterations taken. If spatial orientation strategy is applied, the initial guess reduces residual for 13 correction equations out of 17 .

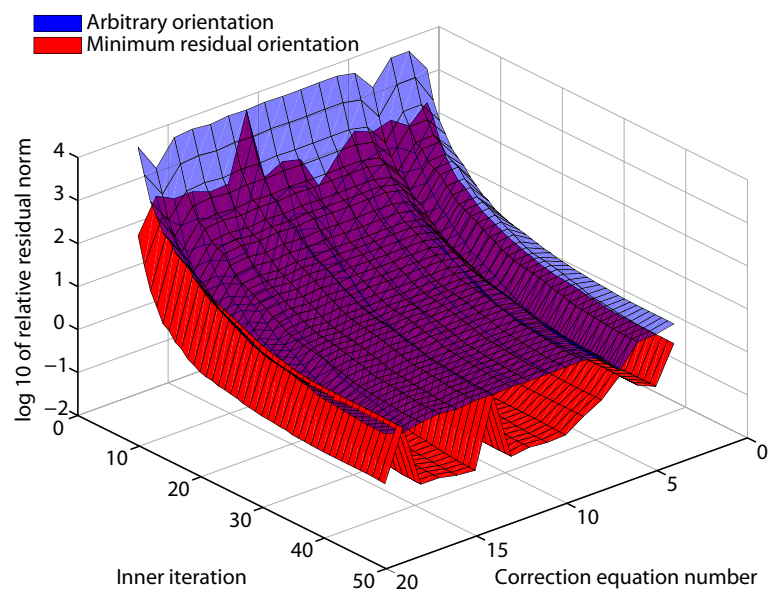

(a)

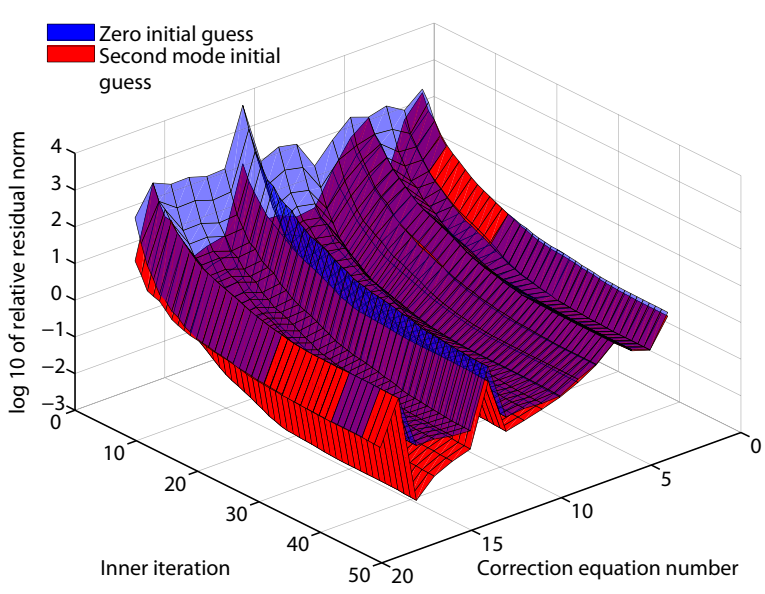

(b)

Figure 7: Effect of spatial orientation (a) and initial guess vector with spatially oriented modes (b) on preconditioned GMRES relative residual convergence history for the test case model in $34-36 \mathrm{kHz}$ region. Faster on average GMRES convergence can be observed in both cases. 
that the perturbation of an eigenvector corresponding to a multiple eigenvalue will only be unstable in the eigenspace corresponding to this multiple eigenvalue. This fact is also corroborated in [18], where the authors have studied the modal interaction of closely spaced natural modes undergoing perturbation and concluded that the amount of interaction depends on the closeness of natural frequencies. Hence we may very well expect that the orthogonal correction to a nominal eigenvector corresponding to a double eigenvalue, lay, at least in part, in the direction of the second nominal eigenvector corresponding to that eigenvalue. For a single mode we might as well seek the correction in the direction of the closest in spectrum neighbor. Thus we use $\gamma \mathbf{x}_{i+1}$ as the initial guess vector for iterative solver of (10), where the scaling coefficient $\gamma$ is chosen such that $\mathbf{P}^{\mathrm{T}}\left(\widehat{\mathbf{A}}-\lambda_{i} \widehat{\mathbf{B}}\right) \mathbf{P} \gamma \mathbf{x}_{i+1}$ is orthogonal to the new residual $\left(\mathbf{P}^{\mathrm{T}}\left(\widehat{\mathbf{A}}-\lambda_{i} \widehat{\mathbf{B}}\right) \mathbf{P} \gamma \mathbf{x}_{i+1}\right)-\mathbf{P}^{\mathrm{T}} \mathbf{r}_{i}$ :

$$
\gamma=\frac{\left(\mathbf{P}^{\mathrm{T}}\left(\widehat{\mathbf{A}}-\lambda_{i} \widehat{\mathbf{B}}\right) \mathbf{P} \mathbf{x}_{i+1}\right)^{\mathrm{T}} \mathbf{P}^{\mathrm{T}} \mathbf{r}_{i}}{\left(\mathbf{P}^{\mathrm{T}} \mathbf{r}_{i}\right)^{\mathrm{T}} \mathbf{P}^{\mathrm{T}} \mathbf{r}_{i}}
$$

This strategy has worked well in practice, note the significantly lower initial residual depicted in Fig. 5 and relative convergence history after 40 iterations of preconditioned GMRES shown in Fig. 7(b).

\subsubsection{Preconditioning}

In order to accelerate the convergence of iterative sparse linear systems solvers, an auxiliary linear system is solved, which is termed preconditioning. Application of right and left preconditioning schemes to Jacobi-Davidson correction equation is well covered in $[8,19,15]$. In particular, an approximation $\mathbf{K}$ of $\left(\widehat{\mathbf{A}}-\lambda_{i} \widehat{\mathbf{B}}\right)$ is used as a preconditioner as long as it is inverted $\widehat{\mathbf{B}}$-orthogonally to the selected subspace. Therefore we apply the projected preconditioner matrix $\left(\mathbf{I}-\mathbf{Q} \mathbf{Q}^{\mathrm{T}} \widehat{\mathbf{B}}\right)^{\mathrm{T}} \mathbf{K}\left(\mathbf{I}-\mathbf{Q} \mathbf{Q}^{\mathrm{T}} \widehat{\mathbf{B}}\right)$, while the associated linear system to be solved can be written as:

$$
\left(\mathbf{I}-\mathbf{Q} \mathbf{Q}^{\mathrm{T}} \widehat{\mathbf{B}}\right)^{\mathrm{T}} \mathbf{K}\left(\mathbf{I}-\mathbf{Q} \mathbf{Q}^{\mathrm{T}} \widehat{\mathbf{B}}\right) \mathbf{x}=\mathbf{b}
$$

with solution given by [19]:

$$
\mathbf{x}=\left(\mathbf{I}-\mathbf{K}^{-1} \widehat{\mathbf{B}} \mathbf{Q}\left(\mathbf{Q}^{\mathrm{T}} \widehat{\mathbf{B}} \mathbf{K}^{-1} \widehat{\mathbf{B}} \mathbf{Q}\right)^{-1} \mathbf{Q}^{\mathrm{T}} \widehat{\mathbf{B}}\right) \mathbf{K}^{-1} \mathbf{b}
$$

provided that $\mathbf{Q}^{\mathrm{T}} \widehat{\mathbf{B}} \mathbf{K}^{-1} \widehat{\mathbf{B}} \mathbf{Q}$ is non-singular. Then the Jacobi-Davidson correction equation preconditioned from the right can be solved in two steps:

$$
\begin{aligned}
& \left(\mathbf{I}-\mathbf{Q} \mathbf{Q}^{\mathrm{T}} \widehat{\mathbf{B}}\right)^{\mathrm{T}}\left(\widehat{\mathbf{A}}-\lambda_{i} \widehat{\mathbf{B}}\right)\left(\mathbf{I}-\mathbf{K}^{-1} \widehat{\mathbf{B}} \mathbf{Q}\left(\mathbf{Q}^{\mathrm{T}} \widehat{\mathbf{B}} \mathbf{K}^{-1} \widehat{\mathbf{B}} \mathbf{Q}\right)^{-1} \mathbf{Q}^{\mathrm{T}} \widehat{\mathbf{B}}\right) \mathbf{K}^{-1} \mathbf{y}=-\left(\mathbf{I}-\mathbf{Q} \mathbf{Q}^{\mathrm{T}} \widehat{\mathbf{B}}\right)^{\mathrm{T}} \mathbf{r}_{i} \\
& \Delta \mathbf{x}_{i}=\left(\mathbf{I}-\mathbf{K}^{-1} \widehat{\mathbf{B}} \mathbf{Q}\left(\mathbf{Q}^{\mathrm{T}} \widehat{\mathbf{B}} \mathbf{K}^{-1} \widehat{\mathbf{B}} \mathbf{Q}\right)^{-1} \mathbf{Q}^{\mathrm{T}} \widehat{\mathbf{B}}\right) \mathbf{K}^{-1} \mathbf{y}
\end{aligned}
$$

One of the most obvious choices for a preconditioner is an ILU (Incomplete LU) factorization of $\left(\widehat{\mathbf{A}}-\lambda_{i} \widehat{\mathbf{B}}\right)$. ILU preconditioners have been successful in many general symmetric, indefinite, and nonsymmetric cases [20]. However, as the authors pointed out, if the method is applied to indefinite matrices severe problems can occur, in particular small pivots may lead to unstable inaccurate factorizations as well as the structure of the original matrices may cause unstable triangular solves. The nature of large mistuning problem for periodic systems featuring high modal density and target eigenvalues commonly located in the interior of the spectrum makes $\left(\widehat{\mathbf{A}}-\lambda_{i} \widehat{\mathbf{B}}\right)$ highly indefinite and severely ill conditioned once we are close to a target eigenvalue. As a result, we have not succeeded in computing any computationally attractive reasonable quality ILU preconditioner. Zero pivots and extremely ill conditioned LU factors were identified as sources of errors. These results are in part corroborated by [19], where authors observed the necessity for a great deal of fill-in (number of non-zero entries) in order to get efficient preconditioning matrices for interior eigenvalues. 
As an alternative, we propose to exploit the block-circulant structure of the unperturbed matrix pair (A, B) in combination with SPAI (Sparse Approximate Inverse) algorithm [21]. Recall that a blockcirculant matrix is completely block-diagonalized by the real Fourier matrix:

$$
\underset{h=1}{\operatorname{Bdiag}}\left[\mathbf{A}_{h}-\lambda \mathbf{B}_{h}\right]=(\mathbf{F} \otimes \mathbf{I})(\mathbf{A}-\lambda \mathbf{B})\left(\mathbf{F}^{\mathrm{T}} \otimes \mathbf{I}\right)
$$

where $\otimes$ is matrix Kronecker product, Bdiag [·] denotes a pseudo-block diagonal matrix, where the $h=1, \ldots, \mathrm{H}$

size of a block changes according to harmonic $h$, and the real Fourier matrix given in [2] is defined as:

$$
\mathbf{F}=\frac{1}{\sqrt{\mathrm{N}}}\left[\begin{array}{ccccc}
1 & \sqrt{2} & 0 & \cdots & 1 \\
1 & \sqrt{2} \cos \alpha & \sqrt{2} \sin \alpha & \cdots & -1 \\
1 & \sqrt{2} \cos 2 \alpha & \sqrt{2} \sin 2 \alpha & \cdots & 1 \\
\vdots & \vdots & \vdots & \ddots & \vdots \\
1 & \sqrt{2} \cos (\mathrm{N}-1) \alpha & \sqrt{2} \sin (\mathrm{N}-1) \alpha & \cdots & (-1)^{\mathrm{N}-1}
\end{array}\right]
$$

$\alpha=2 \pi / \mathrm{N}$ denotes the interblade phase angle. We propose to approximate the inverse of each block $\left(\mathbf{A}_{h}-\lambda \mathbf{B}_{h}\right)^{-1}$ with SPAI algorithm because of its robustness and stability, as compared to ILU. It follows that the application of preconditioner to a vector $\mathbf{x}$ can be carried out by the following steps:

1. $\mathbf{f}=\operatorname{DFT}(\mathbf{x})$

2. $\mathbf{f}=\operatorname{SPAI}\left(\operatorname{Bdiag}\left[\mathbf{A}_{h}-\lambda \mathbf{B}_{h}\right]\right) \mathbf{f}$

3. $\mathbf{y}=\operatorname{IDFT}(\mathbf{f})$

First we decompose $\mathbf{x}$ as a linear combination of the real Fourier basis vectors through DFT (Discrete Fourier Transform), then the real block-Fourier coefficients contained in $\mathbf{f}$ are multiplied by sparse approximation of the inverse of harmonic blocks $\left(\mathbf{A}_{h}-\lambda \mathbf{B}_{h}\right)^{-1}$ before they are reassembled by the IDFT (Inverse Discrete Fourier Transform) to produce the output vector $\mathbf{y}$. The DFT can be implemented efficiently by making use of the fast Fourier transform algorithm with reduced computational complexity. The following set of experiments shows the effect of applying several preconditioners, in particular ILU (0), ILUT and DFT-SPAI, on the convergence behavior of GMRES solver after 40 iterations have been taken. Figs. 8(a) and 8(b) show that blind application of incomplete factorization is rather unsuccessful as compared to DFT-SPAI preconditioning. In case of structure-based ILU (0) we observe greater inaccuracy due to dropping nonzeros, while unstable triangular solves that may have been caused by very small pivots in addition to $(\mathbf{L U})^{-1}$ high condition number are the main reason of ILUT failure, note in Tab. 1 that a relatively large fill-in is done in vain. Apart from SPAI

\begin{tabular}{rr}
$\begin{array}{r}\text { Preconditioner } \\
\text { type }\end{array}$ & $\begin{array}{r}\text { Number of non } \\
\text { zero entries }\end{array}$ \\
\hline ILU(0) & 8474496 \\
ILUT & 71805692 \\
DFT-SPAI & 8461279 \\
\hline
\end{tabular}

Table 1: Comparison of fill-in in applied preconditioners

robustness, its success can be explained by a very high fill-in of the transformed back from Fourier domain matrix $\left(\mathbf{F}^{\mathrm{T}} \otimes \mathbf{I}\right) \mathbf{S P A I}\left(\right.$ Bdiag $\left.\left[\mathbf{A}_{h}-\lambda \mathbf{B}_{h}\right]\right)(\mathbf{F} \otimes \mathbf{I})$. Note also that the DFT-SPAI preconditioner is $h=1, \ldots, \mathrm{H}$ 


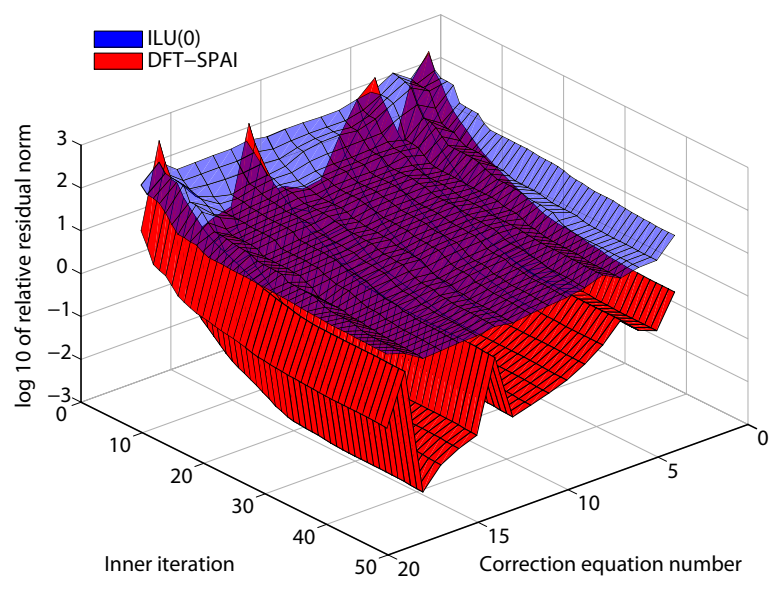

(a)

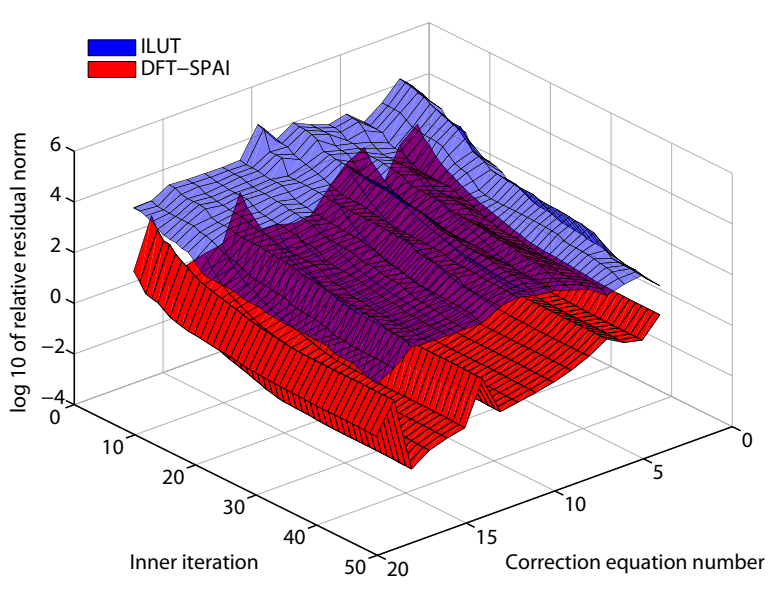

(b)

Figure 8: Comparison of GMRES relative residual convergence history with ILU (0) and DFT-SPAI (a), ILUT and DFT-SPAI (b) preconditioners for the test case model in $34-36 \mathrm{kHz}$ region. The DFT-SPAI preconditioner consistently outperforms both structure-based and threshold-based ILU.

independent of perturbation and can be precalculated off-line, while ILUT uses very large amounts of non-zero entries with additional storage requirements and computational effort for factorization each time perturbation changes, thus putting severe limitations on the maximum problem size that can be handled.

\subsection{Algorithm description}

The Jacobi-Davidson method applied to geometric mistuning problem of periodic systems that implements the computational strategies described in the previous sections is outlined in Algorithm 1. Before applying the main algorithm, a set of preconditioner matrices has to be computed not only with nominal eigenvalues, but also with target values equally covering the frequency band of ROM to have a high-quality preconditioner in case if any localized mode falls in that part of spectrum. The procedure starts with a block of $m$ initial nominal eigenvectors and expands the basis by a block of $k$ vectors. These vectors are approximate solutions of $k$ correction equations, each for one member of double mode with lower residual norm. Note that a more restrictive correction equation (15) is implemented, where we are looking for a correction in the space $\widehat{\mathbf{B}}$-orthogonal to the subspace spanned by all selected eigenvectors, each is a member of double mode with smaller residual. This approach, suggested in [9], leads to faster convergence in addition to better conditioned linear correction systems in presence of clustered eigenvalues.

As with all iterative inner-outer processes, we must carefully evaluate the overall computational cost balancing inner solves precision and number of outer iterations. If the correction equations are solved exactly, we may often achieve convergence for all approximated eigenpairs after first RayleighRitz projection, yet solving inner system to high precision may be very slow and costly. It is generally acknowledged that if a Krylov subspace method is used as linear iterative solver the residual will not decrease substantially up until a large enough subspace is built containing significant spectral information corresponding to omitted parts of the spectrum, which may be prohibitive for very large order systems. Therefore a suitable inner stopping condition scheme must be applied to avoid any superfluous work while computing accurate solutions whenever useful.

As suggested in the literature and based on our experimental observations, the block methods that solve all linear correction equations simultaneously do not consistently improve the overall run- 


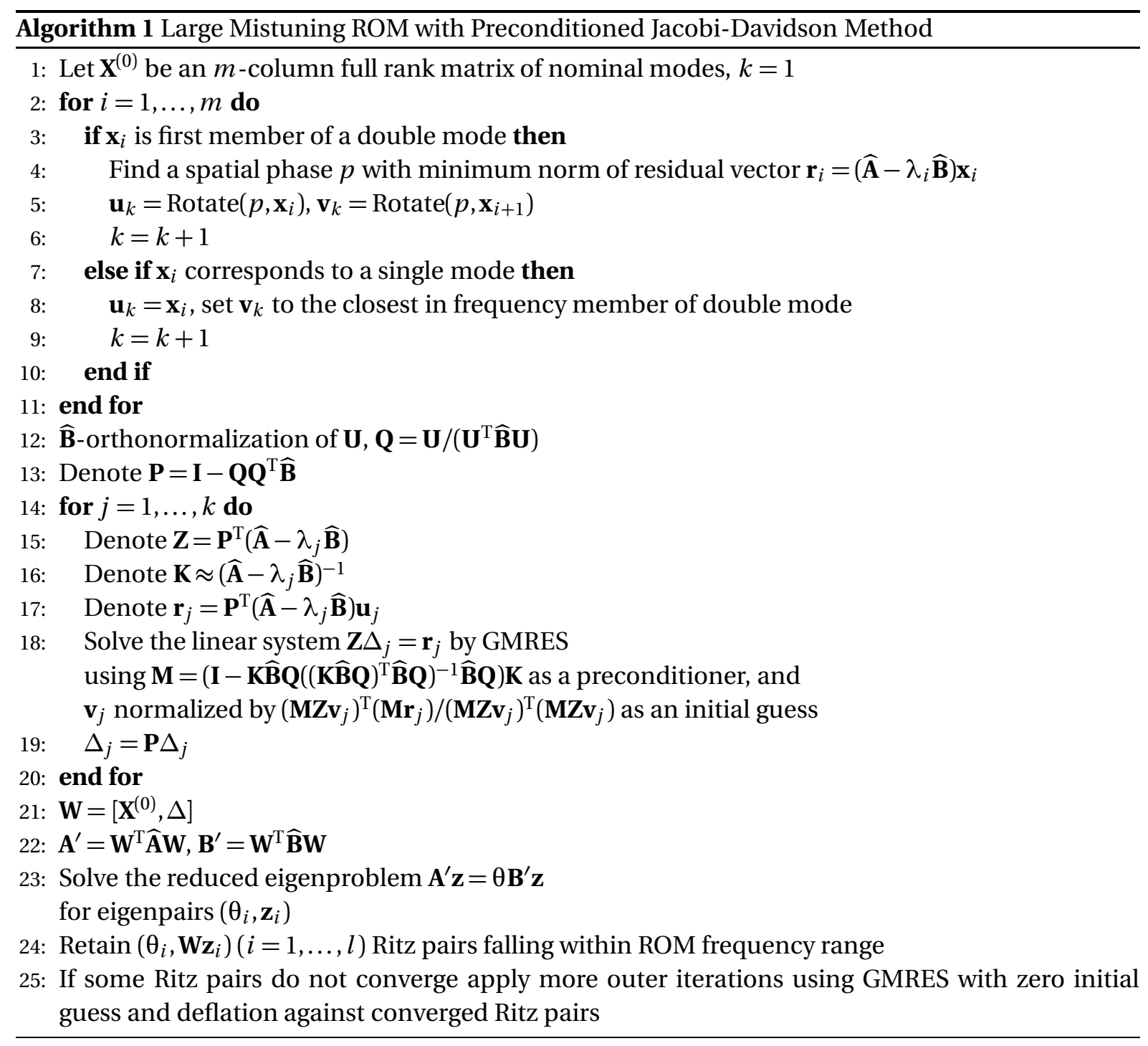


time, unless the eigenpairs are multiple or highly clustered. Therefore from the point of view of performance and efficiency, provided that after first outer iteration the non-converged eigenpairs do not cluster, we choose to solve one correction equation per outer iteration. Moreover, because of the different rates of convergence of each of the approximate eigenvectors, we perform explicit deflation by including them in $\mathbf{Q}$, however, they still have to be present in the basis of the trial subspace of the Rayleigh-Ritz method. After each outer step we may have a number of non-converged eigenpairs in the selected area of spectrum. While only one will be drawn for correction, there is a choice to be made. The most common targeting schemes are: to always select the non-converged eigenvector with eigenvalue closest to a target and to select the one with minimum residual. The rationale for latter is that the selected Ritz pair with minimum residual would converge first to be removed from the following iterations. In the proposed implementation the minimum residual targeting is adopted in order to avoid selecting a spurious Ritz pair, which may be a linear combination of eigenpairs far to the left and to the right of the targeted spectrum.

\section{Numerical studies}

In this section we present a few numerical experiments. We are mainly interested in assessing the speed and accuracy of SMC and JD algorithms in typical situations. The results obtained by ROM will be compared with those of the full FEM reference model of the mistuned bladed disk. Only free response results are computed because if we neglect the modal truncation error, the accuracy of the solution to the forced response problem at resonance frequencies is fully determined by the errors in the approximated natural frequencies and mode shapes. The frequency bands of $34-36 \mathrm{kHz}$ and $15-16 \mathrm{kHz}$ corresponding to the blade motion dominated mode families are chosen for analysis and marked by horizontal lines ( $2 \mathrm{~S}$ and $2 \mathrm{~T} / 2 \mathrm{~F}$ mode families correspondingly) in Fig. 2. Their selection is motivated by the fact that they represent two typical situations. In particular, the higher frequency family $2 \mathrm{~S}$ spans a larger frequency range. The frequency veerings can be observed in that region causing some modes belonging to different families to interact. As the result of generally higher modal density in that area there is less separation between mode families. In fact, as we mentioned earlier, for symmetrical systems the sensitivity of an eigenvector to perturbation depends on the separation of its corresponding eigenvalue from other eigenvalues. Therefore $34-36 \mathrm{kHz}$ region represents a more difficult to approximate case under equivalent perturbation than that of $15-16 \mathrm{kHz}$, or $2 \mathrm{~T} / 2 \mathrm{~F}$ mode family, which is well separated from others, in addition to spanning a narrower frequency range.

In our first example we look for 29 perturbed eigenpairs in $15-16 \mathrm{kHz}$ region. Figs. 9(a) and 9(b) present nominal and perturbed natural frequencies along with the canonical angles between corresponding eigenvectors. Note the presence of a strongly affected by perturbation eigenpair with eigenvalue separated from the rest of cluster and eigenvector making large angle with all nominal eigenvectors that corresponds to a highly localized mode depicted in Fig. 10(a). Figs. 11(a) and 11(b) show the natural frequency errors on a logarithmic scale and MAC ratio between perturbed mode shapes predicted by SMC and reference model ANSYS modal analysis for the $15-16 \mathrm{kHz}$ frequency band calculated with $f_{c}=15,400 \mathrm{~Hz}$, arbitrarily chosen in the middle of the frequency band. The results show that for this case of relatively narrow well isolated family of modes the correction term calculated with $f_{c}=15,400 \mathrm{~Hz}$ is still accurate enough to yield approximation for the highly localized $14,965 \mathrm{~Hz}$ mode. Thus natural frequency error for all calculated modes is below $0.003 \%$ and MAC value above 0.9995 . Next we apply our new iterative method for the same problem. We solve the correction equations approximately by using the Matlab built-in GMRES method gmres.m, that showed faster convergence on the test case system. We have precalculated a set of SPAI preconditioners with target value covering the frequency region $15-16 \mathrm{kHz}$ with a step $\sim 100 \mathrm{~Hz}$. For all the numerical tests the maximum inner iteration number is set to 500 , while the inner iteration tolerance varies. In 


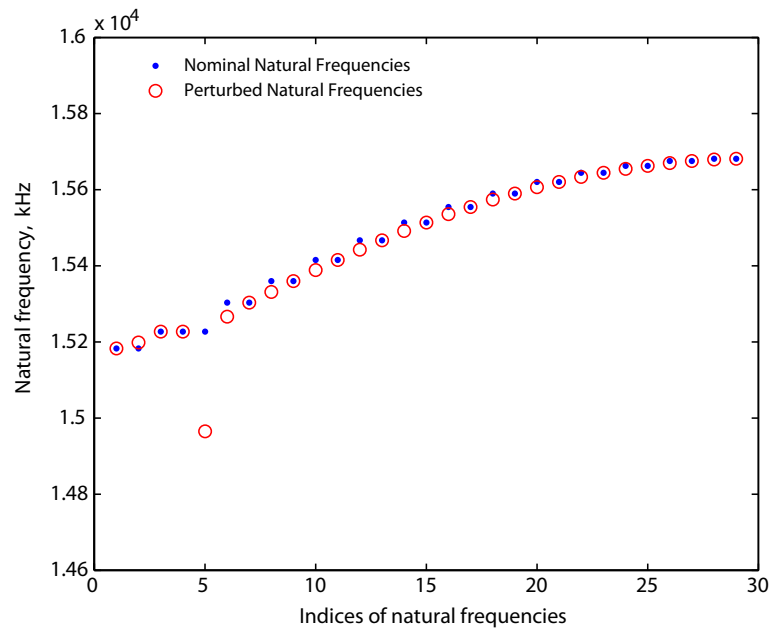

(a)

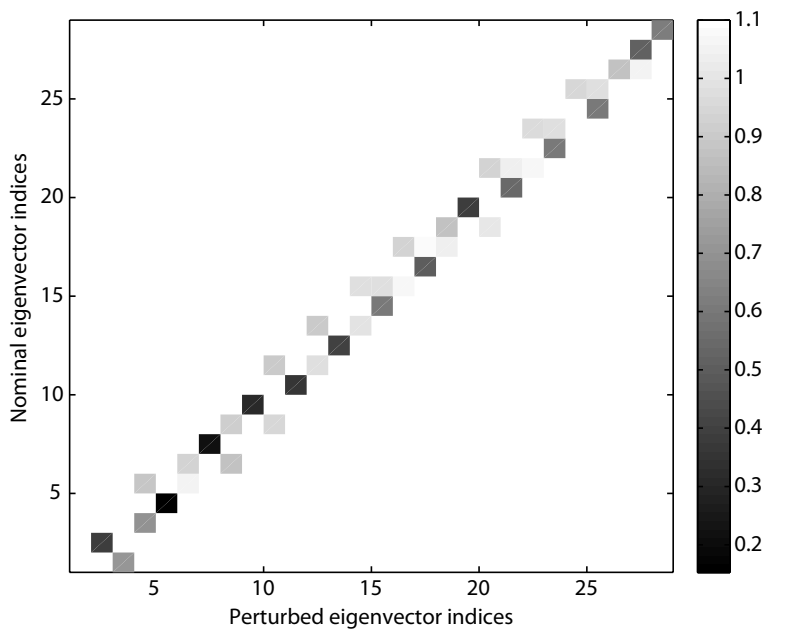

(b)

Figure 9: Natural frequencies (a) and canonical angles between eigenvectors (b) of nominal and perturbed test case model in $15-16 \mathrm{kHz}$ region. The "rogue" localized mode can be seen with natural frequency far away from the original cluster and large angle with nominal ones in the lower left corner of plot (b).

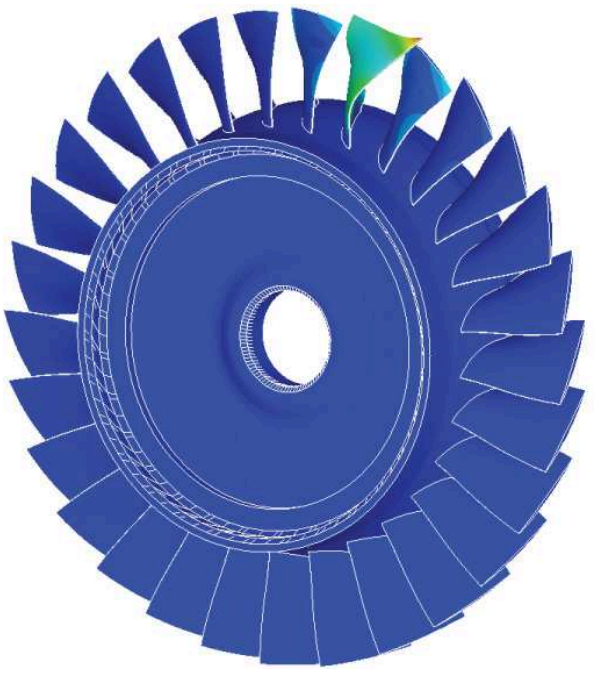

(a)

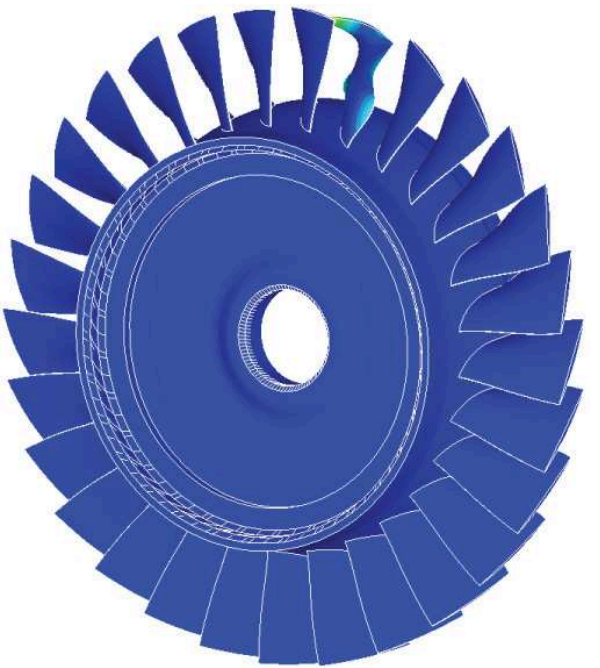

(b)

Figure 10: Localized mode shapes corresponding to 14,965 Hz (a) and 33,940 Hz (b). 


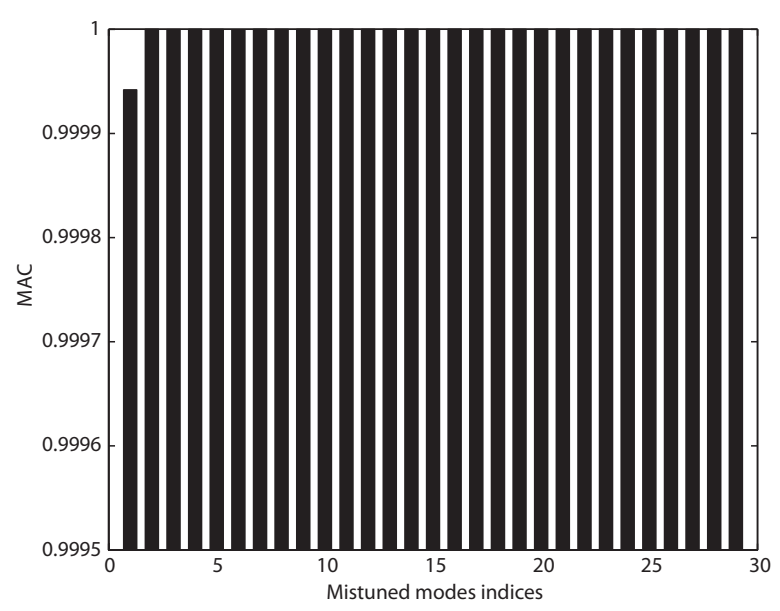

(a)

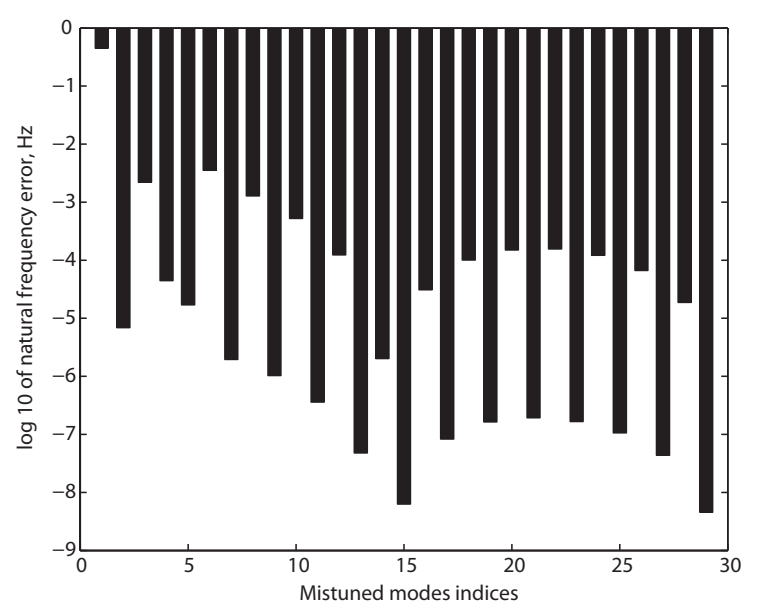

(b)

Figure 11: MAC ratio (a) and natural frequency error (b) between reference and approximated by SMC eigenpairs for the test case model in $15-16 \mathrm{kHz}$ region. SMC accurately approximates perturbed eigenpairs in this region, with MAC above 0.9995 and natural frequency error below $0.003 \%$.

Fig. 12 we show the effect of increasing inner solver accuracy on MAC, $\log 10$ of natural frequency error and number of GMRES iterations for each of the correction equations solved used as a measure of the computational cost. Note that a reasonably accurate estimate of the perturbed eigenpairs can be obtained without applying any further outer iterations, provided that the inner correction equations are solved accurately enough. Apparently with 500 steps GMRES and inner relative residual tolerance set to $4 \cdot 10^{-10}$ the correction equations are solved to high enough precision to yield good quality correction terms, and the results are in line with the literature that suggests to apply more accurate inner solves if near convergence. In the current investigation we try to obtain the highest reasonable precision and stop if reach plateau in GMRES convergence. The performance of the method should be tailored by taking into account the overall running time, which in parallel computing environment suggests the strategy of decreasing the number of outer iterations by applying more accurate inner solves. Next we carry out a more severe test to the large geometric mistuning ROM algorithms, that features higher modal density with frequency veering regions corresponding to $2 \mathrm{~S}$ family of modes. The effect of perturbation on nominal modes in that area is shown in Figs. 3 and 4(a), while Figs. 13(a) and 13(b) depict the natural frequency errors and MAC ratio in the $34-36 \mathrm{kHz}$ frequency band calculated by SMC with $f_{c}=34,700 \mathrm{kHz}$. Clearly in this case the highly localized mode shown in Fig. 10(b) is not properly approximated, with the error in natural frequency more that $0.73 \%$ and MAC ratio 0.86 . The error could be attributed to the choice of the centering frequency being far from the mode that needs a high quality correction. This example illustrates one of the weak points of SMC method discussed above, i.e. SMC algorithm may not be an optimal choice if applied in the wider areas with high modal density even for local low rank perturbations. Now we consider again the $34-36 \mathrm{kHz}$ region and seek 33 perturbed eigenpairs with the iterative method. Here we apply the inner solver settings that yielded acceptable precision in the previous experiment, namely maximum of $500 \mathrm{GM}-$ RES iterations with the inner relative residual tolerance set to $4 \cdot 10^{-10}$. After solving 17 correction equations to the selected accuracy level, we find that 11 of approximate eigenparairs do not converge to the outer residual norm tolerance set to $9 \cdot 10^{5}$. Fig. 14 reports the residual convergence history of those eigenpairs that have not converged after first outer iteration, while in Tab. 2 the computational cost is provided in terms of number of GMRES solves. Note that even if only one correction equation is solved per outer iteration with the selected targeting strategy, we sometimes observe the convergence of several eigenpairs at a time. The peaks in the convergence behavior of certain eigenpairs 


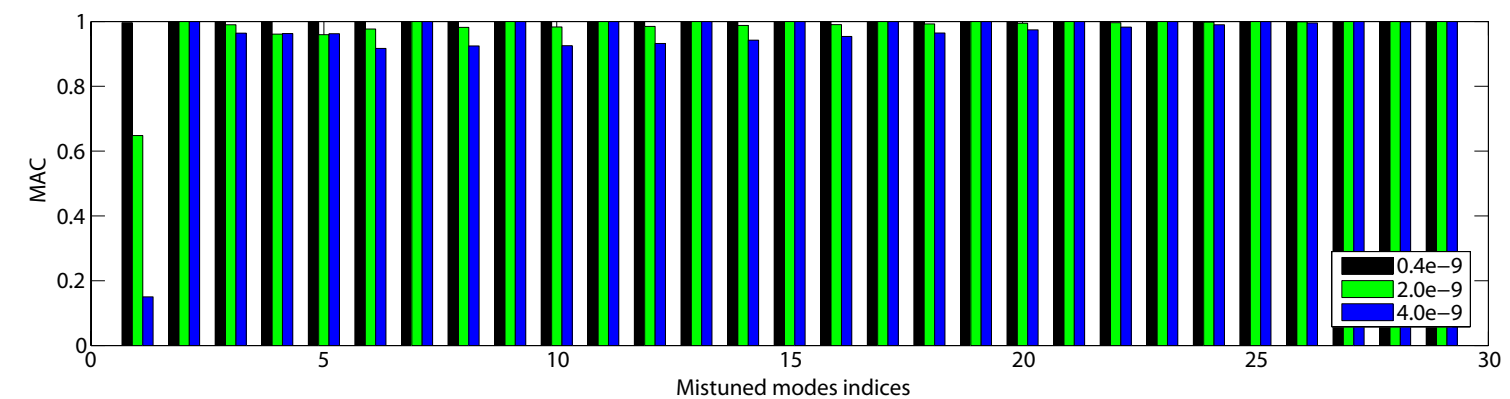

(a)

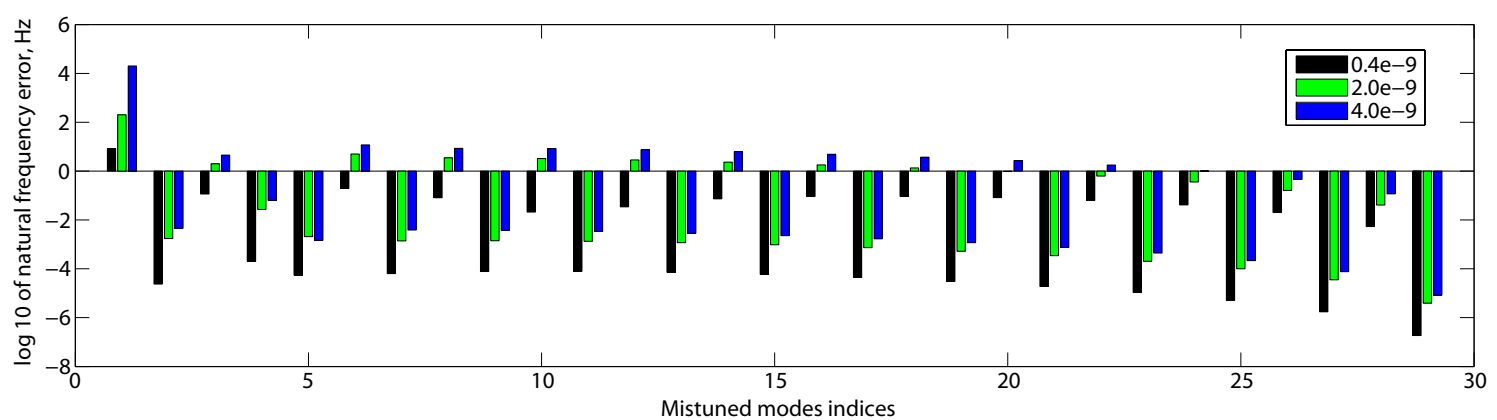

(b)

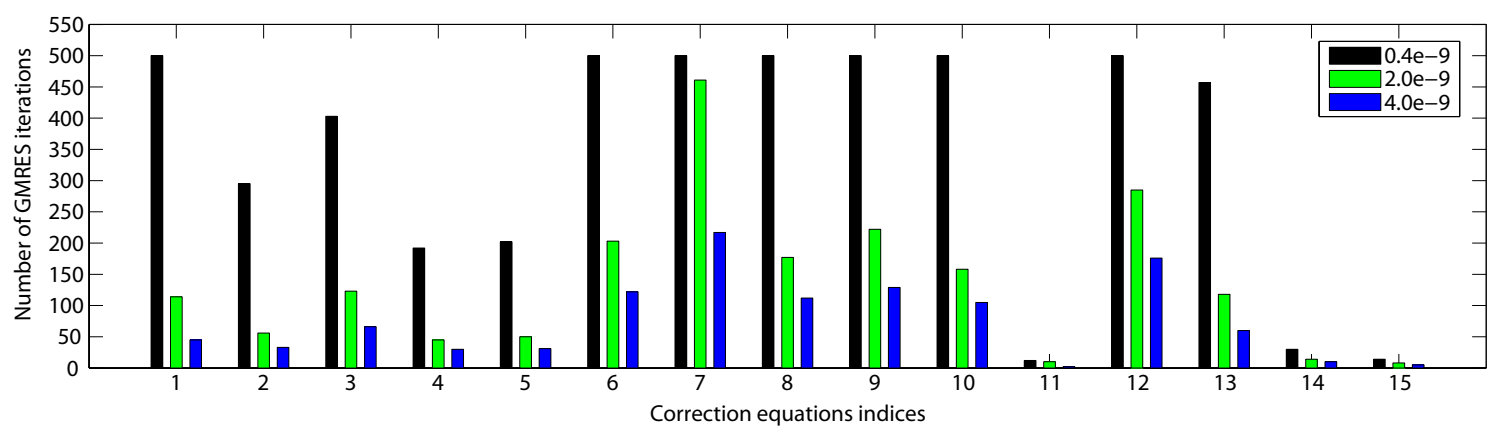

(c)

Figure 12: Effect of GMRES relative residual tolerance on MAC (a), natural frequency error (b) and number of inner solves (c) for the test case model in $15-16 \mathrm{kHz}$ region. A reasonable quality solution can be obtained with a single outer iteration by increasing the inner solver accuracy. With a total of 5101 GMRES iterations taken the MAC for all modes is above 0.996 and natural frequency error below $0.05 \%$. 


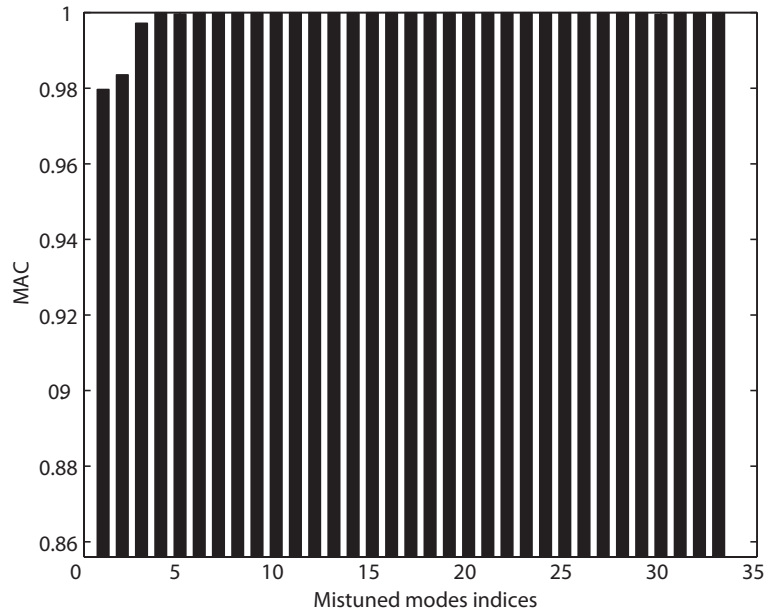

(a)

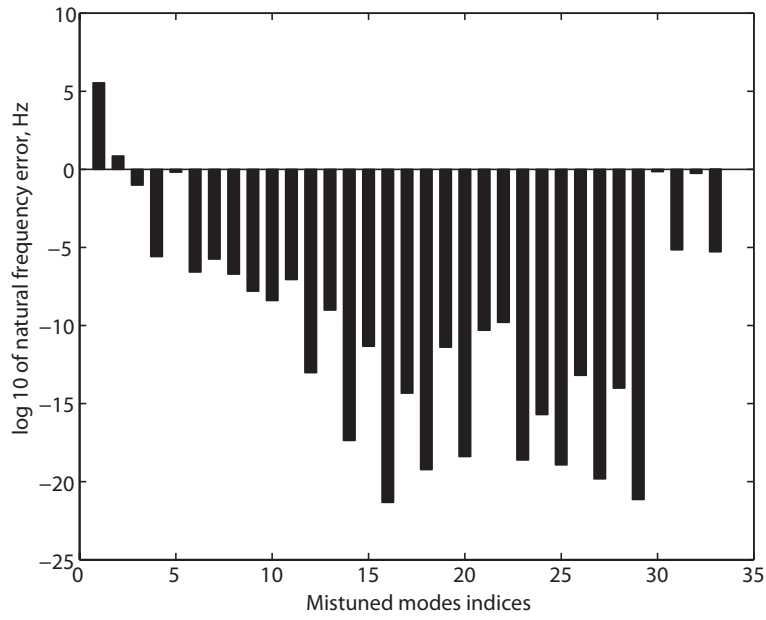

(b)

Figure 13: MAC ratio (a) and natural frequency error (b) between reference and approximated by SMC eigenpairs for the test case model in $34-36 \mathrm{kHz}$ region. In this case SMC fails to accurately approximate localized perturbed mode corresponding to $33,940 \mathrm{~Hz}$, which has MAC 0.86 and natural frequency error $0.73 \%$ due to poor preconditioning calculated with $f_{c}=34,700 \mathrm{kHz}$.

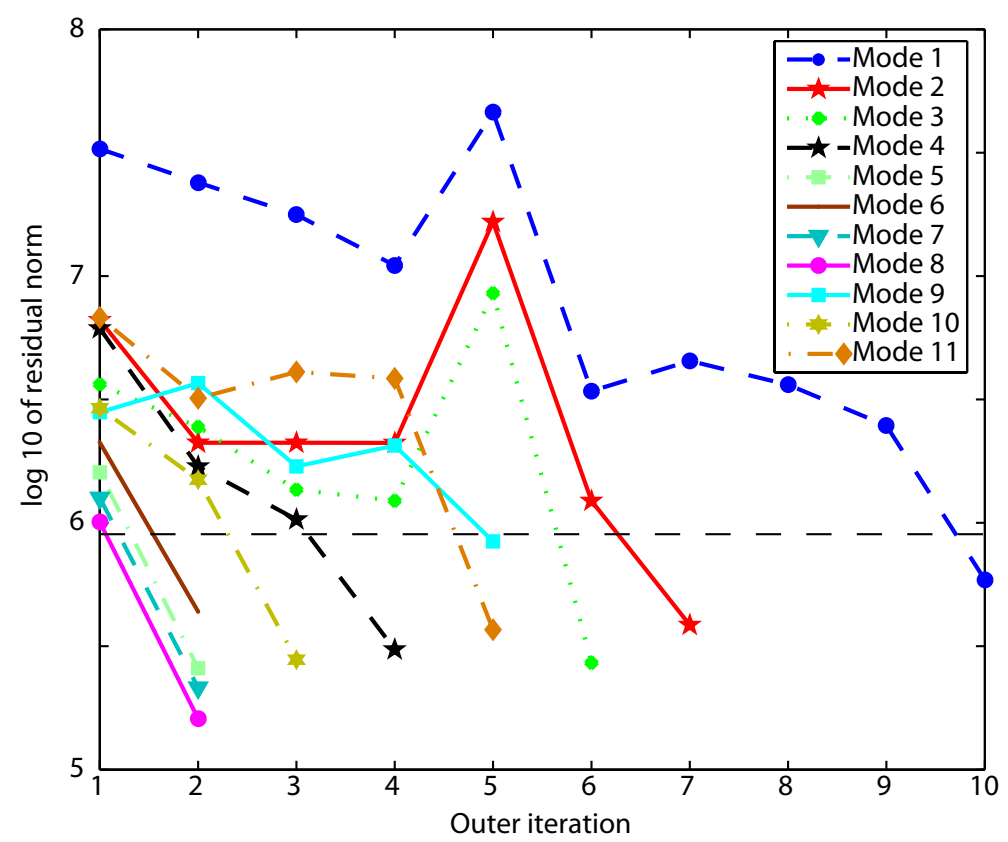

Figure 14: Outer loop convergence history of 11 modes after first outer iteration of the preconditioned iterative method in $34-36 \mathrm{kHz}$ region. Each curve shows the convergence of the residual norm of a Ritz pair fallen in $33.9-35.2 \mathrm{kHz}$ region at outer steps. Note that the outer residual tolerance level is marked by the dashed horizontal line.

are likely to be caused by unstable convergence to internal eigenspaces, which is technically possible but not guaranteed by the theory. The accuracy of approximate eigenpairs after applying 10 outer iterations are presented in Figs. 15(a) and 15(b).

Summarizing this section, these two examples demonstrate the claim from Section 2 that SMC may not yield an accurate approximation when nominal modes selected for ROM are spread over 


\begin{tabular}{rrr}
\hline $\begin{array}{r}\text { Outer } \\
\text { iteration }\end{array}$ & $\begin{array}{r}\text { Number of } \\
\text { GMRES } \\
\text { iterations }\end{array}$ & $\begin{array}{r}\text { Number of } \\
\text { converged } \\
\text { modes }\end{array}$ \\
\hline 1 & 7354 & 22 \\
2 & 500 & 4 \\
3 & 500 & 1 \\
4 & 500 & 1 \\
5 & 500 & 2 \\
6 & 500 & 1 \\
7 & 500 & 1 \\
8 & 500 & 0 \\
9 & 500 & 0 \\
10 & 500 & 1 \\
\hline Total & 11854 & 33 \\
\hline
\end{tabular}

Table 2: Computational cost and number of converged eigenpairs per outer iteration

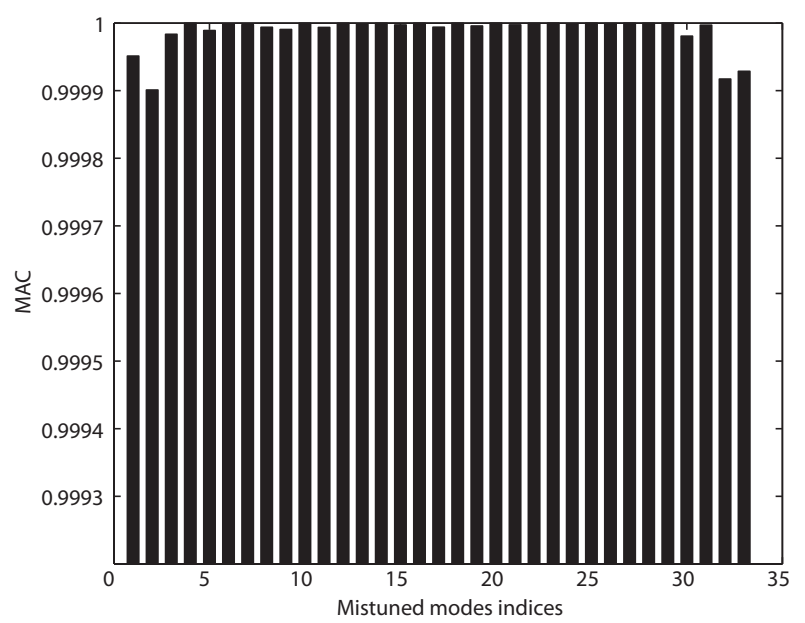

(a)

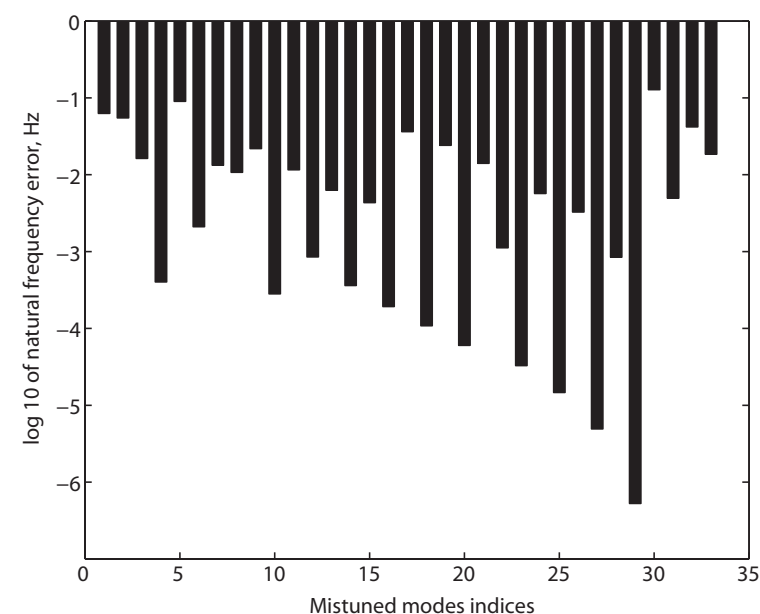

(b)

Figure 15: MAC ratio (a) and natural frequency error (b) between reference and approximated by the preconditioned iterative method eigenpairs for the test case model in $34-36 \mathrm{kHz}$ region. MAC ratio for all modes after 10 outer iterations taken is above 0.9992 and natural frequency error below $0.0002 \%$.

wider areas of spectrum, i.e. in the modal interaction areas, while the iterative preconditioned method consistently produces an accurate reduced-order model. Considering the cases where both methods provide reasonable approximation quality, the preference should be given based on computational time and the ability to scale to larger order industrial models. Since in our numerical experiments we use the industrial code of SMC implemented in FORTRAN and only Matlab prototype of the iterative method, it is difficult to compare performance in terms of actual computational time. The algorithmic complexity of SMC depends to a larger extend on the rank of the perturbation (the number of mistuned DOF $m$ ) and the order of nominal system $n$. Essentially, it has the complexity $\mathscr{O}\left(\mathrm{m}^{3}\right)$, which mainly accounts for the derivation of compensated basis vectors that incurs the solution of dense linear system of the order $m$ in equation (6) of [10] and a few BLAS3 operations of the same 
order. The storage requirement roughly amounts to $\mathscr{O}(\mathrm{nm})$ that makes its scaling to larger order models practically infeasible.

Estimation of the computational cost of preconditioned iterative method is a more delicate issue because it concerns the stopping criterion for the inner solver, whose analysis is still an active area of research. Many factors should be considered before setting the optimal condition (cost of preconditioning, relative cost of inner versus outer iterations, etc.). The performance of such methods is usually evaluated by taking into account the cost of matrix-vector multiplications, which is the most time consuming computation. Since (A, B) are sparse, the computational cost per one matrixvector operation will depend on the type of sparsity. But the overall complexity will be essentially proportional to the total number of inner iterations. Therefore if we insure optimal/suboptimal convergence by restricting admissible perturbations it will likely to grow linearly in $n$. As in any iterative method, the memory requirements are also limited as $n$ grows, they amount to storing one sparse block of $(\mathbf{A}, \mathbf{B})$, its perturbation and a few vectors of order $n$. Both methods incur some off-line computational effort. A set of nominal quasi-static modes is computed for SMC, those are dense blocks and therefore dependent of the structure of perturbation to be memory efficient. On the contrary, the new method requires a set of sparse preconditioners, which are completely independent of perturbation. It is likely that neither of the methods is a clear winner; SMC may be more efficient for medium size models, still the detailed complexity analysis based on experimental observations made with both FORTRAN implementations is needed to evaluate the relative performance.

\section{Conclusion}

This paper addresses the problem of quantifying and predicting forced response of geometrically mistuned rotors by building compact ROM in a computationally efficient way. First we have analyzed the behavior of SMC method revealing that it is closely related to the generalized Davidson algorithm. There are all indications that from the memory efficiency and accuracy point of view it is a good choice for the moderate order FEM models under low rank localized perturbation if narrow clustered areas of spectrum are analyzed. For very large scale industrial models as well as for the areas of spectrum where multiple mode families interact, a new method is proposed. It stems from the Jacobi-Davidson algorithm implementing a number of simple heuristic strategies based on the block-circulant structure of the nominal system and assumptions on perturbation. In particular, a number of typical industrial applications of ROM are considered such as manufacturing imprecision, erosion or foreign objects damage event that constitute rather local low rank high amplitude perturbation destroying symmetry. A set of numerical experiments have been conducted on an industrial bladed disk model. In its current simple form the implementation of the method demonstrates promising numerical results. Our experience indicates that the algorithm combined with proposed preconditioning scheme routinely converges to the perturbed interior eigenspace within reasonable time, provided that the perturbation is localized to a few sectors. However this work is preliminary, and more research is needed, both to further develop the method and to study the extension of these results to the case of high rank low magnitude perturbation that cannot be effectively handled by existing "small mistuning" ROM. On the other hand, the new method may replace or complement the SMC in the algorithm proposed in [10] to study the combined effect of "large" deterministic mistuning and "small" random mistuning on bladed disks.

\section{References}

[1] M. Castanier, G. Ottarsson and C. Pierre, A reduced-order modeling technique for mistuned bladed disks, Journal of Vibration and Accustics 119(3) (1997) 439-447. 
[2] R. Bladh, M. Castanier, C. Pierre and M. Kruse, Dynamic response predictions for a mistuned industrial turbomachinery rotor using reduced order modeling, ASME Journal of Engineering for Gas Turbines and Power 124(2) (2002) 311-324.

[3] M. Yang and J. Griffin, A reduced order model of mistuning using a subset of nominal system modes, ASME Journal of Engineering for Gas Turbines and Power 123 (2001) 893-900.

[4] S. Lim, R. Bladh, M. Castanier and C. Pierre, A compact, generalized component mode mistuning representation for modeling bladed disk vibration, AIAA Journal 45(9) (2007) 2285-2298.

[5] S. Lim, M. Castanier and C. Pierre, Vibration modeling of bladed disks subject to geometric mistuning and design changes, in: Proceedings of the 45-th AIAA/ASME/ASCE/AHS/ASC Structures, Structural Dynamics and Material Conference, paper 2004-1686, Palm Springs, California, USA, 2004.

[6] A. Sinha, Reduced-order model of a bladed rotor with geometric mistuning, in: Proceedings of ASME Turbo Expo 2007: Power for Land, Sea and Air, paper GT2007-27276, Montreal, Quebec, Canada, 2007.

[7] E. Petrov, K. Sanliturk and D. Ewins, A new method for dynamic analysis of mistuned bladed disks based on the exact relationship between tuned and mistuned systems, ASME Journal of Engineering for Gas Turbines and Power 124 (2002) 586-597.

[8] G. Sleijpen, A. Booten and D. Fokkema, Jacobi-Davidson type methods for generalized eigenproblems and polynomial eigenproblems, BIT Numerical Mathematics 36(3) (1996) 595-633.

[9] A. Sameh and Z. Tong, The trace minimization method for the symmetric generalized eigenvalue problem, SIAM Journal of Computational and Applied Mathematics 123 (2000) 155-175.

[10] V. Ganine, M. Legrand, H. Michalska and C. Pierre, A reduction technique for mistuned bladed disks with superposition of large geometric mistuning and small model uncertainties, in: The 12-th International Symposium on Transport Phenomena and Dynamics of Rotating Machinery, paper 2008-20158, Honolulu, Hawaii, USA, 2008.

[11] A. Stathopoulos, Nearly optimal preconditioned methods for hermitian eigenproblems under limited memory. Part I: Seeking one eigenvalue, SIAM Journal on Scientific Computing 29(2) (2007) 481-514.

[12] J. Lombard, J. Dupeux, C. Dupont, F. Garcin and S. Baumhauer, Mistuning phenomena on bladed disk: Industrial methods and applications, in: Proceedings of ASME Turbo Expo 2006: Power for Land, Sea and Air, paper GT2006-90205, Barcelona, Spain, 2006.

[13] O. Bendiksen, Localization phenomena in structural dynamics, Chaos, Solutions and Fractals 11(10) (2000) 1621-1660.

[14] M. Kim, J. Moon and J. Wickert, Spatial modulation of repeated vibration modes in rotationally periodic structures, ASME Journal of Vibration and Acoustics 122(1) (2000) 62-68.

[15] D. Fokkema, L. Sleijpen and H. Van der Vorst, Jacobi-Davidson style QR and QZ algorithms for the reduction of matrix pencils, SIAM Journal on Scientific Computing 20(1) (1998) 94-125.

[16] G. Stewart and J. Sun, Matrux Perturbation Theory, Academic Press, San Diego CA, 1990.

[17] T. Zhang, G. Golub and K. Law, Subspace iterative methods for eigenvalue problems, Linear Algebra and its Applications 294 (1999) 239-258. 
[18] M. Yang and J. Griffin, A normalized modal eigenvalue approach for resolving modal interation, ASME Journal of Engineering for Gas Turbines and Power 119 (1997) 647-650.

[19] G. Sleijpen, H. Van Der Vorst and E. Meijerink, Efficient expansion of subspaces in the JacobiDavidson methods for standard and generalized eigenproblems, Electronic Transactions on Numerical Analysis 7 (1998) 75-89.

[20] C. Edmond and Y. Saad, Experimental study of ILU preconditioners for indefinite matrices, SIAM Journal of Computational and Applied Mathematics 86 (1997) 387-414.

[21] M. Grote and T. Huckle, Parallel preconditioning with sparse approximate inverses, SIAM Journal on Scientific Computing 18 (1997) 838-853. 